\title{
Quasar variability: Correlations with amplitude
}

\author{
M.R.S. Hawkins \\ Institute for Astronomy (IfA), University of Edinburgh, Royal Observatory, Blackford Hill Edinburgh EH9 3HJ, Scotland \\ e-mail: mrsh@roe.ac.uk
}

Received July 6, 1998; accepted February 15, 2000

\begin{abstract}
The relation between quasar variability and parameters such as luminosity and redshift has been a matter of hot debate over the last few years with many papers on the subject. Any correlations which can be established will have a profound effect on models of quasar structure and evolution. The sample of quasars with redshifts in ESO/SERC field 287 contains over 600 quasars in the range $0<z<3.5$ and is now large enough to bin in luminosity and redshift, and give definitive measures of the correlations. We find no significant correlation between amplitude and redshift, except perhaps at very low redshift, but an inverse correlation between amplitude and luminosity. This is examined in the context of various models for quasar variability.
\end{abstract}

Key words: quasars: general

\section{Introduction}

One of the most important constraints on the structure of quasars is variability. Short term variations set limits on the size of the emitting region, and differences in the nature of the variation in the X-ray, optical and radio domains give clues to the underlying structure. Variations on longer timescales of a few years are not so easily accounted for by the current black hole paradigm, and microlensing has been put forward as an alternative explanation for the observations. Although the most extensive monitoring of quasar variability has been done in optical wavebands, there is still little consensus even over the broad picture. One of the first problems is to parametrise the variation in such a way that it can be compared with models, or even more general expectations. The timescale of variation and the amplitude are the two parameters which have been mostly studied, although some authors have succeeded in confusing the two. This typically involves claiming that in a short run of data, objects varying on a short timescale will achieve a larger amplitude than those varying on a longer timescale, and so amplitude can be taken as an (inverse) measure of timescale (Hook et al. 1994).

In this paper we shall confine our attention to the correlation of amplitude with other parameters. Amplitude is an easy parameter to measure, and involves none of the difficulties inherent in estimating timescale of variation. There are modes of variability in which some uncertainty will be introduced by the length of the run of observations, but this is a problem which can arise in any time series analysis. Most attention has so far been given to possible correlations of amplitude with redshift or luminosity (e.g. Trevese et al. 1989; Giallongo et al. 1991; Hook et al. 1994; Cristiani et al. 1996), but even in such straightforward situations there has been little agreement.

The reasons for the lack of consensus are not easy to understand, but it is clear that any effect which is present is not large compared with the cosmic scatter in the data, which in the case of amplitude appears to be about one magnitude, much larger than any photometric errors. Perhaps the most likely cause of disagreement is in the selection of samples for analysis. In order to obtain a meaningful measure of correlation it is essential that unbiassed samples are used, and that they cover a large range of redshift and luminosity. In this paper particular attention is paid to the sample selection process, and by extending the analysis to high redshifts the baseline for the measurement of correlations is greatly extended over earlier samples.

\section{Quasar samples}

\subsection{Observational material}

The parent sample of quasars for the analysis of variability amplitude comes from a large scale survey and monitoring programme being undertaken in the ESO/SERC field 287 , at $21 \mathrm{~h} 28 \mathrm{~m},-45^{\circ}$. Quasars have been selected according to a number of criteria, including colour, variability, radio emission and objective prism spectra, or 
a combination of these techniques. Redshifts for over 600 quasars have so far been obtained, and several complete samples defined within specific limits of magnitude, redshift and position on the sky (Hawkins \& Véron 1995). A detailed description of the survey is given by Hawkins \& Véron (1995) and Hawkins (1996). Briefly, a large set of UK 1.2 Schmidt plates spanning 20 years was scanned with the COSMOS and SuperCOSMOS measuring machines to provide a catologue of some 200000 objects in the central 19 square degrees of the plate. These were calibrated with CCD frames to provide light curves in $B_{\mathrm{J}}$ and $R$, and colours in $U B V R I$. Photometric errors have been discussed in detail in earlier papers (Hawkins 1996 and references therein) and are $\sim 0.08$ magnitudes for any individual machine measurement, and only weakly dependent on magnitude. There are approximately four plates in each year which reduces the error to $\sim 0.04$ magnitudes per epoch. This is small compared with the amplitudes of interest in this paper, and no attempt has been made to deconvolve it.

\subsection{Sample selection}

For the analysis in this paper three samples will be defined. The first (UVX) is based only on position on the sky and ultraviolet excess. The area on the sky containing the sample is defined by a number of AAT AUTOFIB fields and a $2 \mathrm{dF}$ field (Folkes et al. 1999 and references therein), covering a total area of 7.0 square degrees. Within this area all objects with $U-B<-0.2$ and $B_{\mathrm{J}}<21.0$ were observed. This was extended to $B_{\mathrm{J}}<21.5$ in the $2 \mathrm{dF}$ field. The ultra-violet excess (UVX) cut although necessary to give a relatively clean sample of quasar candidates, has the well known limitation of only being effective for redshifts $z<2.2$. Beyond this redshift quasars become red in $U-B$ as the Lyman forest enters the $U$ band. For samples at higher redshift, variability has been found to provide a very useful criterion for quasar selection (Hawkins 1996). The second sample for consideration here (VAR), was selected on this basis, with the requirement that the object should lie in the same area of sky as for the first sample with a magnitude limit $B_{\mathrm{J}}<21$, or anywhere in the measured area of the plate with a magnitude limit $B_{\mathrm{J}}<19.5$, and should have an amplitude $\delta m>0.35$. The defining epoch for the magnitudes was the year 1977. This sample was selected without any reference to colour and so can be used to measure trends over a large range of redshift $(z<3.5)$. The third sample (AMP) was selected in a similar manner, but over the whole measured area of the plate (19 square degrees), and with an amplitude cut $\delta m>1.1$. There is some evidence that these large amplitude objects form a distinct group, which is discussed below.

The development of fibre fed spectrographs has meant that every object included within a given set of search criteria can be observed to give a high completeness level. In the case of fields observed with AUTOFIB, the existence of forbidden regions in the 40 arcmin field, and the very variable throughput for different fibres aligned at different positions on the spectrograph slit meant that up to $20 \%$ of spectra did not have sufficient signal to provide an unambiguous redshift. These objects where re-observed later with the faint object spectrograph EFOSC on the ESO $3.6 \mathrm{~m}$ telescope at La Silla. The $2 \mathrm{dF}$ observations where of very uniform quality, and the redshift measures or other classification were almost $100 \%$ successful from the fibrefeed spectra.

Details of all three samples, containing a total of 384 quasars, are given in the Appendix. $B$ magnitudes are in the $B_{\mathrm{J}}$ system defined by the IIIa-J emulsion and the GG395 filter, and refer to the year 1977. The amplitude $\delta m$ is the difference between maximum and minmum magnitude achieved over the 20 year run of data. The samples which each quasar belongs to are indicated by 1,2 and 3 corresponding to VAR, UVX and AMP respectively, as defined above. Data for many of the quasars have already been published by Hawkins \& Véron (1995), but are given again here for completeness. Any small differences in the parameters are the result of further refinement of the calibration, and more extended monitoring of the light curves.

UVX selected samples have been used many times in the past for quasar surveys, and the constraints are quite well known. Variability selection has been less often used, and some additional comments are appropriate. In particular, there is the important question of completeness. Hawkins \& Véron (1995) use a small sample of quasars in field 287 from Morris et al. (1991) selected by objective prism to test completeness. In $1993,79 \%$ of the objective prism quasars would have also been detected according to the variability criterion $\delta m>0.35$; by 1997 this figure had risen to $93 \%$, with two quasars remaining below the variability threshold. In fact both of these quasars are clearly variable, with amplitudes 0.32 and 0.29 magnitudes. Strangely, they lie at either extreme of the redshift and luminosity range of the sample, with redshifts $3.23,0.29$ and luminosities $-27.55,-22.33$ respectively. Figure 1a shows the distribution of epoch at which quasars first satisfy the detection criterion given by Hawkins (1996). This is generally speaking equivalent to attaining an amplitude of 0.35 magnitudes. The distribution peaks between 2 and 4 years, and most quasars have satisfied the criterion after 7 years. Figure 1b shows the cumulative distribution of detection epochs, illustrating the point that after 15 years nearly all quasars have varied sufficiently to put them in the variability selected sample.

The distribution of amplitudes is best shown with the UVX sample, which was selected without any reference to variability. Figure $2 \mathrm{a}$ is a histogram of amplitudes over a 20 year baseline, and shows a median amplitude of around 0.7 magnitudes. As would be expected from Fig. 1, nearly all of the sample lies above the 


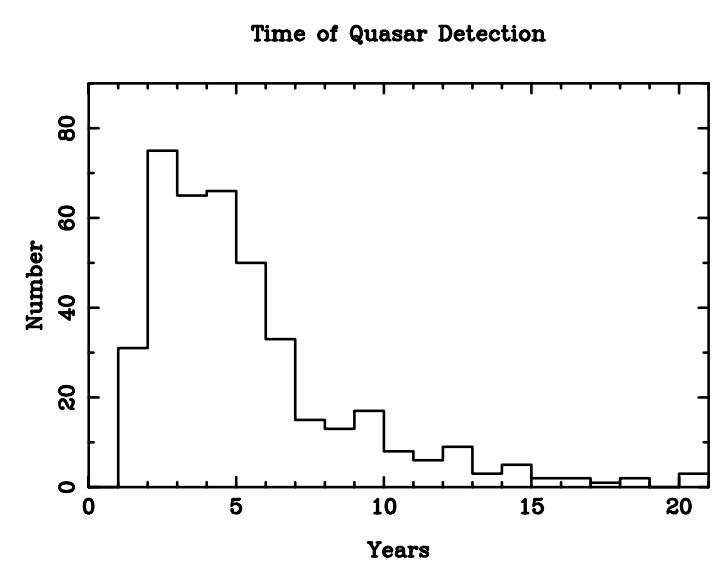

Cumulative Quasar Detection

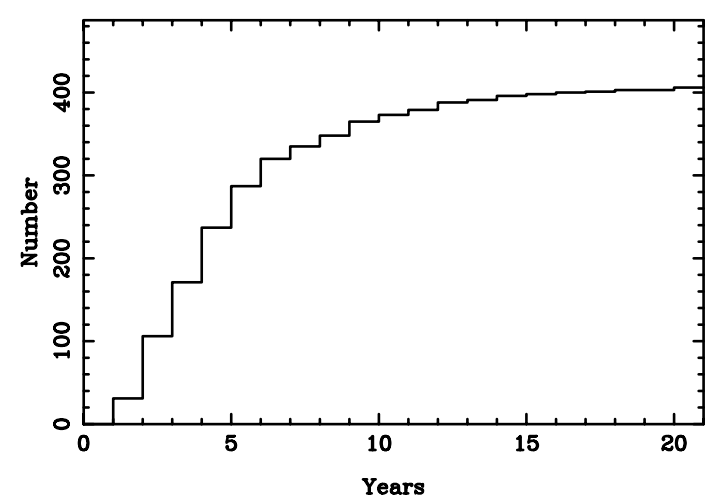

Fig. 1. The top panel shows a histogram of the epochs at which quasars could first have been detected on the basis of the variability criterion. The bottom panel shows the same plot in cumulative form

threshold amplitude of the variability selected sample of 0.35 mags. With this in mind it is worth investigating the possibility of using the variability selected sample to look for correlations at higher redshift. Figure $2 \mathrm{~b}$ shows a histogram of amplitudes for the VAR sample, which from the way it was selected has a cut-off at an amplitude of 0.35 mags. More interestingly, the distribution as a whole peaks at an amplitude of about 0.7 , similar to the UVX sample. This peak is well clear of the cut-off, and implies that only a small fraction of quasars are missed from the VAR sample. There may however be a problem detecting the most luminous quasars, $\left(M_{B}<-27\right)$ for which there is evidence for relatively small variations (Cristiani et al. 1996). Nonetheless, the sample can be used with caution to extend quasar correlations to high redshift.

\section{Amplitude correlations}

The top two panels in Fig. 3 show the distribution of amplitudes as a function of redshift and luminosity for the UVX sample. The most striking thing about these figures is the marked drop in amplitude towards low redshift
$\mathrm{U}-\mathrm{B}<-0.2$
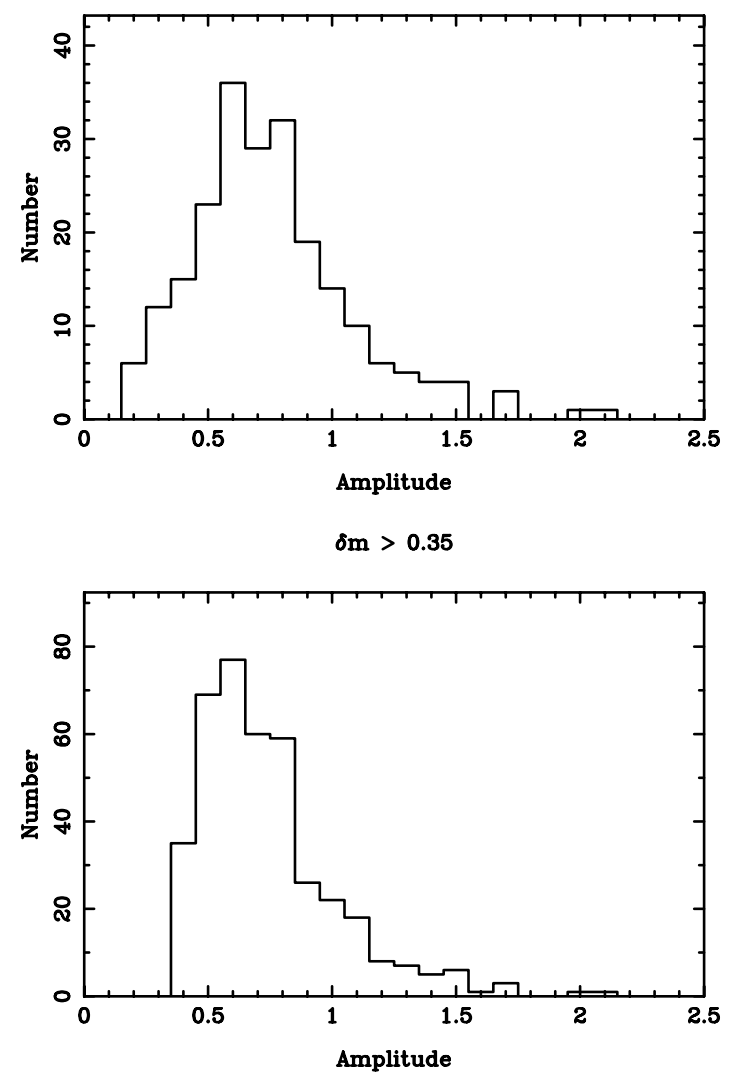

Fig. 2. The top panel shows a histogram of amplitudes for the complete UVX detected sample. The bottom panel shows a similar histogram for the variability detected sample (VAR). Quasars are constrained to have an amplitude greater than 0.35 mags

and luminosity. This is illustrated more clearly in the top two panels of Fig. 4, where the data is binned in intervals of 0.5 in redshift and unit absolute magnitude, and the mean plotted with $\sqrt{N}$ error bars. The fall towards low redshift and luminosity is highly significanti (a 3- $\sigma$ effect), although it is not clear from these data alone whether it is primarily a luminosity or redshift effect. The correlation coeffecient for the top left panel of Fig. 3 with $z<0.5$ is 0.46 , and for the top right panel with $M_{B}>-22$ is -0.67 , confirming the reality of the correlation. There is also some evidence for a decrease in amplitude for the most luminous quasars $\left(M_{B}<-25\right)$, and an even weaker decline for high redshift objects. Correlation coefficients for all the data in the top two panels are 0.12 and -0.10 for left and right panels respectively, emphasising the weakness of the effect. This is another manifestation of the well known degeneracy between redshift and luminosity. This point will be investigated further below by dividing the data into sub-samples.

The bottom two panels in Figs. 3 and 4 show similar plots for the VAR sample. The greater redshift range allowed by variability selection still shows little trend 
$\mathrm{U}-\mathrm{B}<-\mathbf{0 . 2}$

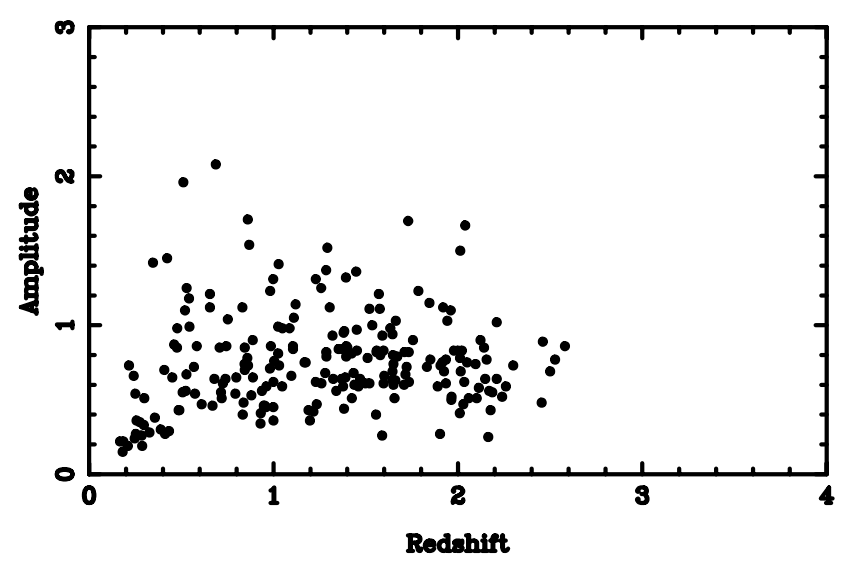

$\delta m>0.35$

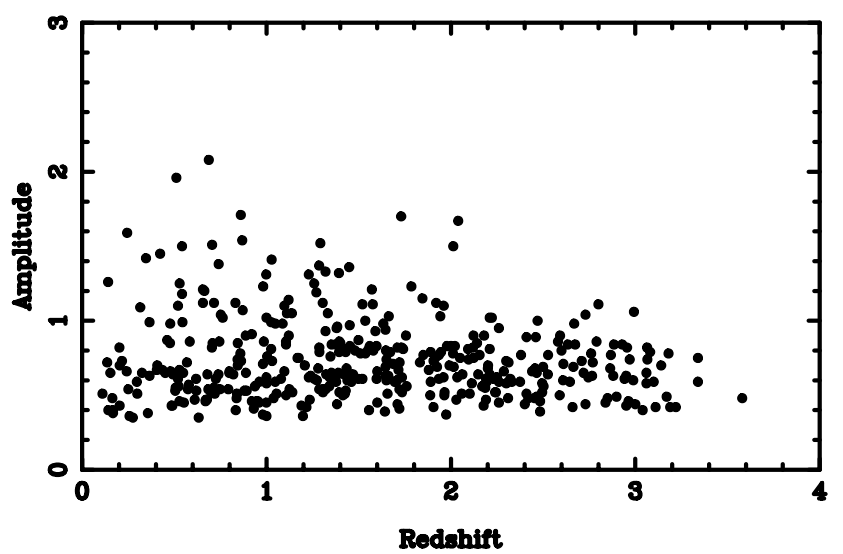

$\mathrm{U}-\mathrm{B}<-\mathbf{0 . 2}$

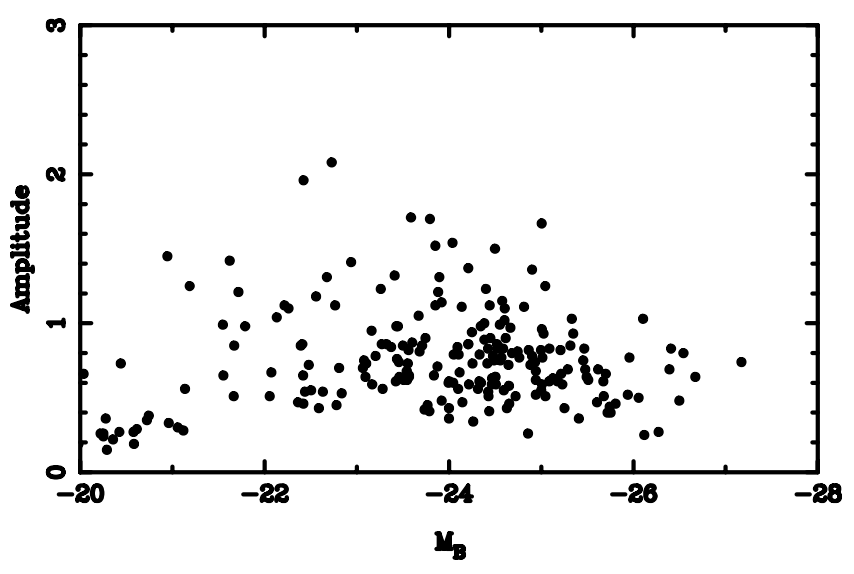

$\delta \mathrm{m}>0.35$

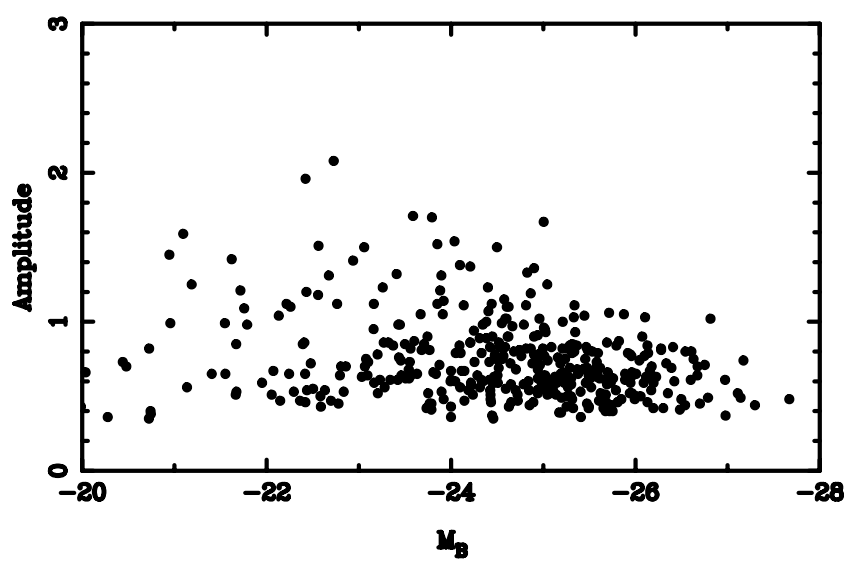

Fig. 3. The top two panels show plots of amplitude versus redshft and absolute magnitude for quasars selected solely on the basis of ultra-violet excess. The bottom two panels are similar plots for quasars selected on the basis of variability

of amplitude with redshift, but the decrease in amplitude for luminous quasars is shown to continue to greater luminosities, although the effect is inevitably lessened by the absence of quasars with $\delta m<0.35$.

It has been mentioned above that there is a degeneracy between redshift and luminosity. This results from the fact that in a magnitude limited sample high redshift quasars tend to be high luminosity objects, and vice versa. Thus a trend with one parameter will be mimicked by a trend with the other, and the true relation will be hard to disentangle. This degeneracy can in principle be broken byi binning the data in redshift and luminosity, and the result of doing this is shown in Figs. 5 and 6 . The VAR sample was used as it covers a wider range of luminosity and redshift, making binning feasible. The left hand panel of Fig. 6 shows amplitude as a function of luminosity in two redshift bins, $z<1.5$ and $z>1.5$. Data for the two redshift ranges overlap nicely, and clearly show a de- crease in the relation between amplitude and luminosity. The right hand panel shows amplitude as a function of redshift in two luminosity bins. In this case there is some evidence for an increase in the relation at low redshift, but it is essentially flat beyond $z=0.5$ for both luminosity ranges. It is however worth noting that the less luminous quasars have larger amplitudes, as expected from the data in the left hand panel. It would thus appear that while for $M_{B}>-25$ amplitude does indeed decrease with luminosity, it does not change with redshift for $z>0.5$.

Examination of Fig. 3, especially the bottom panels, suggests that there may be population of low luminosity and/or low redshift quasars distinct from the parent population. This is particularly evident in the bottom left hand panel of Fig. 3, which is uniformly populated between amplitudes of 1.1 and 1.8 up to a redshift of 2 at which point there is a sharp cut-off with no amplitudes greater than 1.1 at higher redshift. To investigate this population with 
$\mathrm{U}-\mathrm{B}<-0.2$

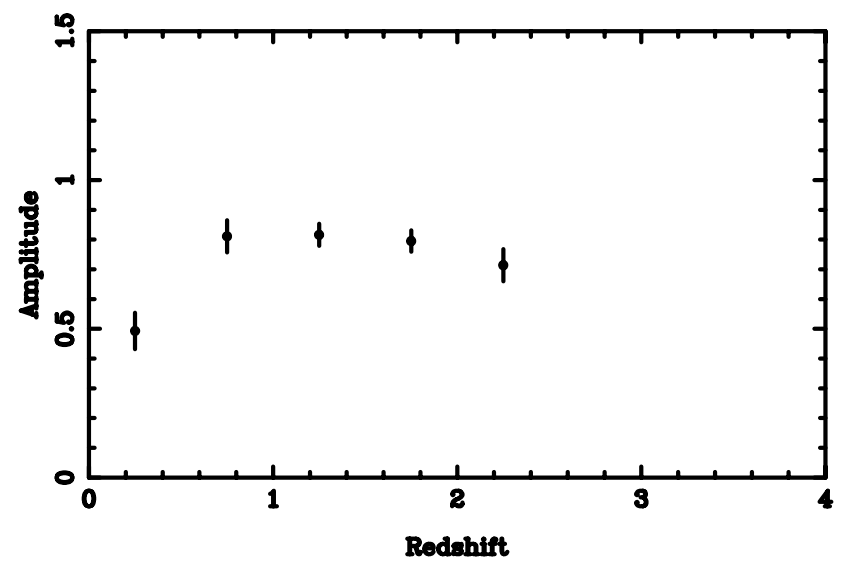

$8 m>0.85$

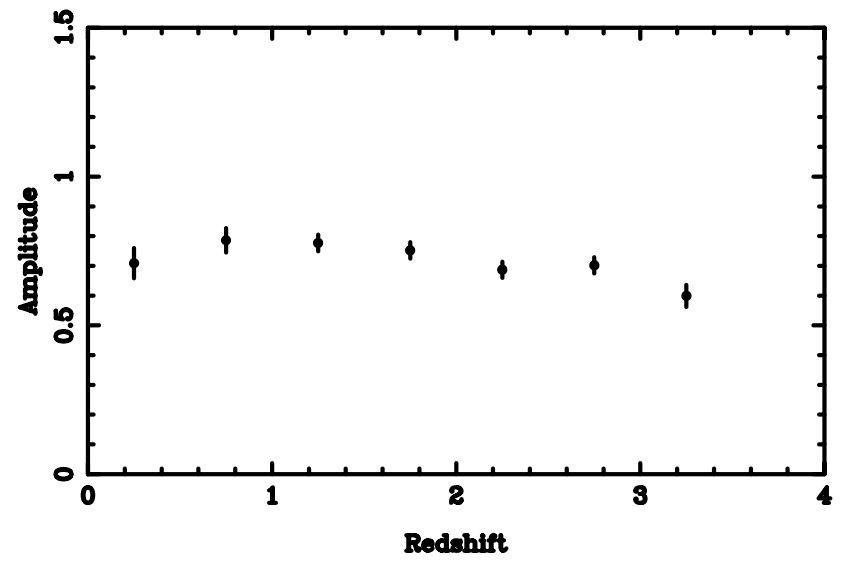

$\mathrm{U}-\mathrm{B}<-0.2$
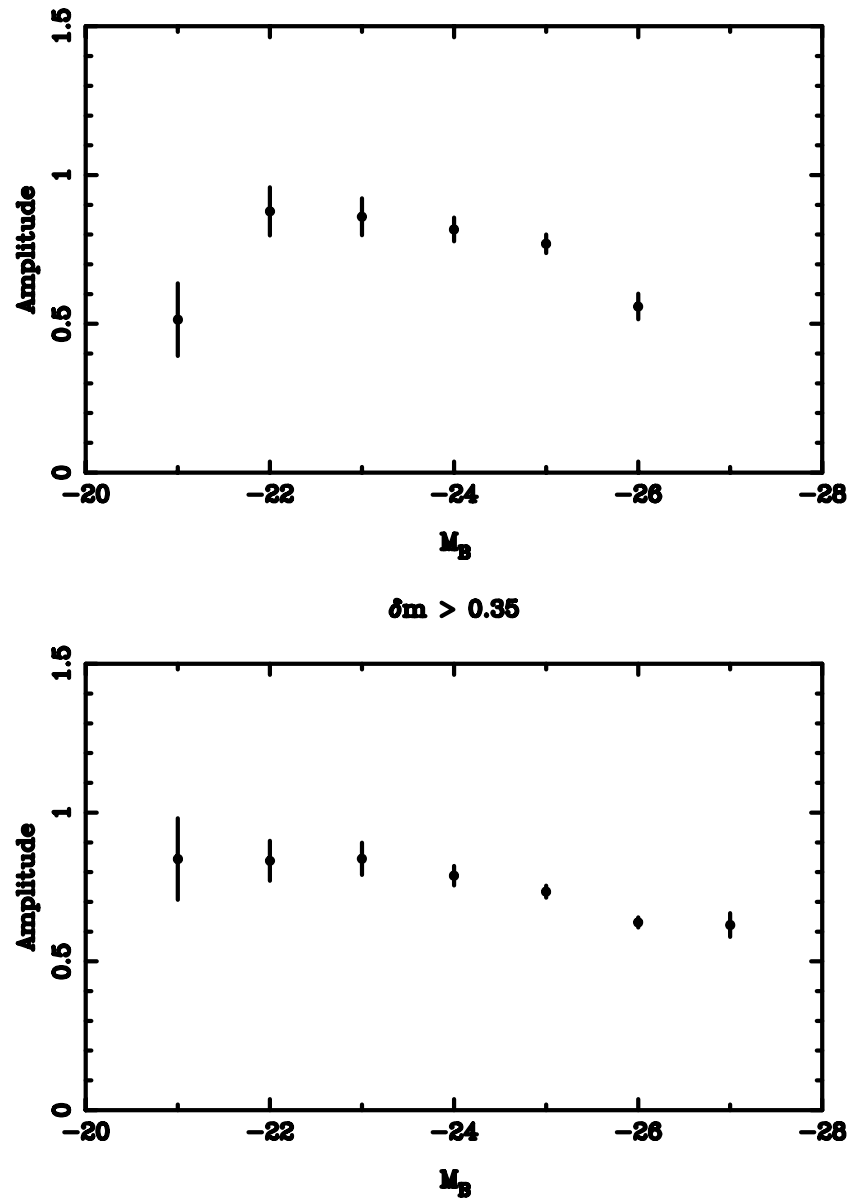

Fig. 4. Plots based on the same data as for Fig. 3, but binned in redshift intervals of 0.5 and unit absolute magnitude intervals. The error bars show the uncertainty in the position of the mean

better statistics all variables with $\delta m>1.1$ in the measured area of field 287 were observed on the $3.6 \mathrm{~m}$ at La Silla to confirm their identification as quasars and measure redshifts. This became the AMP sample. Figure 7 shows amplitude as a function of both redshift and luminosity, and it will be seen that there is indeed a cut-off in redshift at $z \sim 2$ and $M_{B} \sim-25$. It is clear that this cut-off must in fact be related to luminosity. If it were a redshift cut-off there is no reason why such objects should not be seen with greater luminosity. On the other hand if it were a luminosity cut-off, then this combined with a magnitude limit will indeed produce an effective cut-off in redshift.

\section{Discussion}

Attempts to measure correlations of amplitude (or some related variability parameter) with redshift and luminos- ity have a long history, which is summarised by Hawkins (1996). Among recent work, a useful place to start is with the paper by Hook et al. (1994). They analyse a sample of $\sim 300$ quasars in the SGP area from 12 UK $1.2 \mathrm{~m}$ Schmidt plates taken in 5 separate yearly epochs spanning 16 years. They find a convincing anti-correlation between their variability parameter $\sigma_{\mathrm{v}}$ (a measure of variation about the mean) and luminosity, but a much weaker anti-correlation with redshift. They attribute this to the degeneracy between redshift and luminosity in their sample. The same sample is re-analysed by Cid Fernandes et al. (1996) who use a variability index related to variance, and also one related to the structure function. They concur with Hook et al. (1994) that there is an anti-correlation between their variability indices and luminosity, but claim a positive correlation with redshift. This effect is not apparent to the eye, but is interpreted as a variability-wavelength dependence rather than an intrinsic variability-redshift 
$0.0<z<1.5$
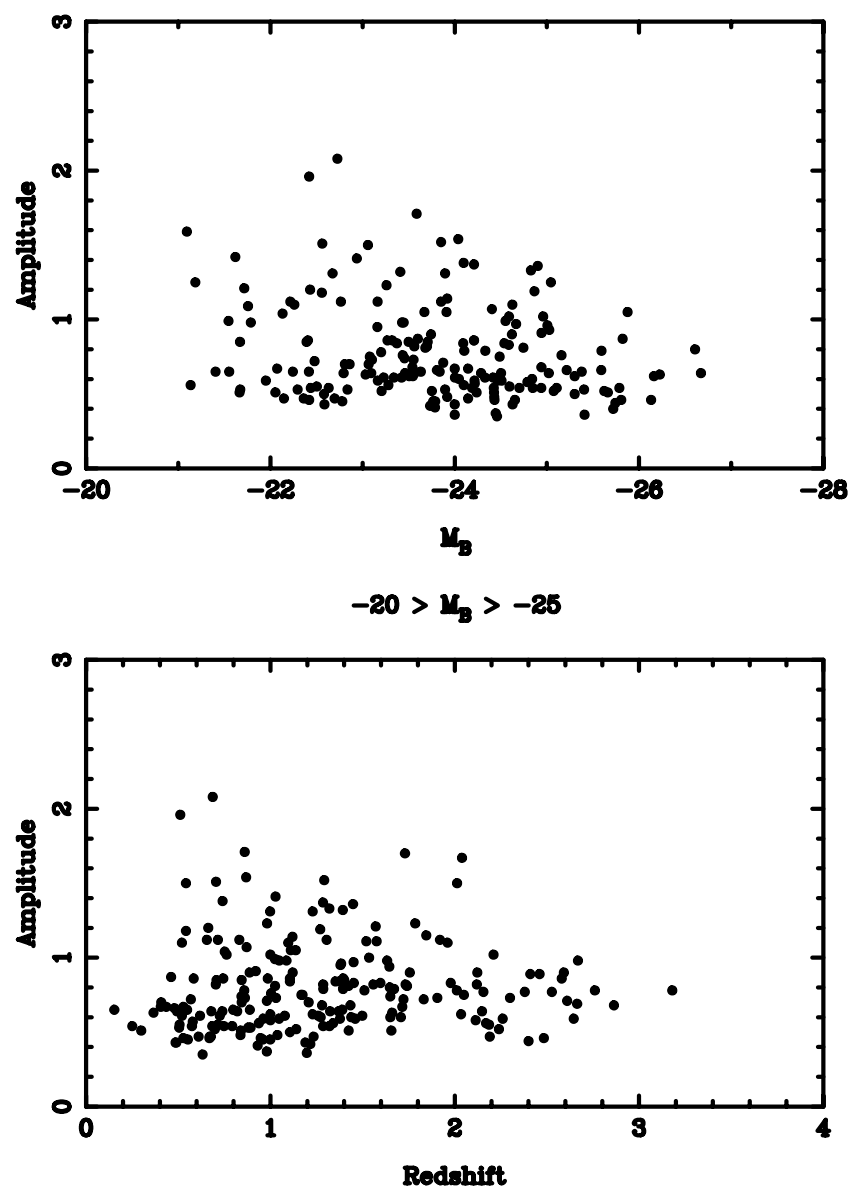

$1.5<z<3.5$
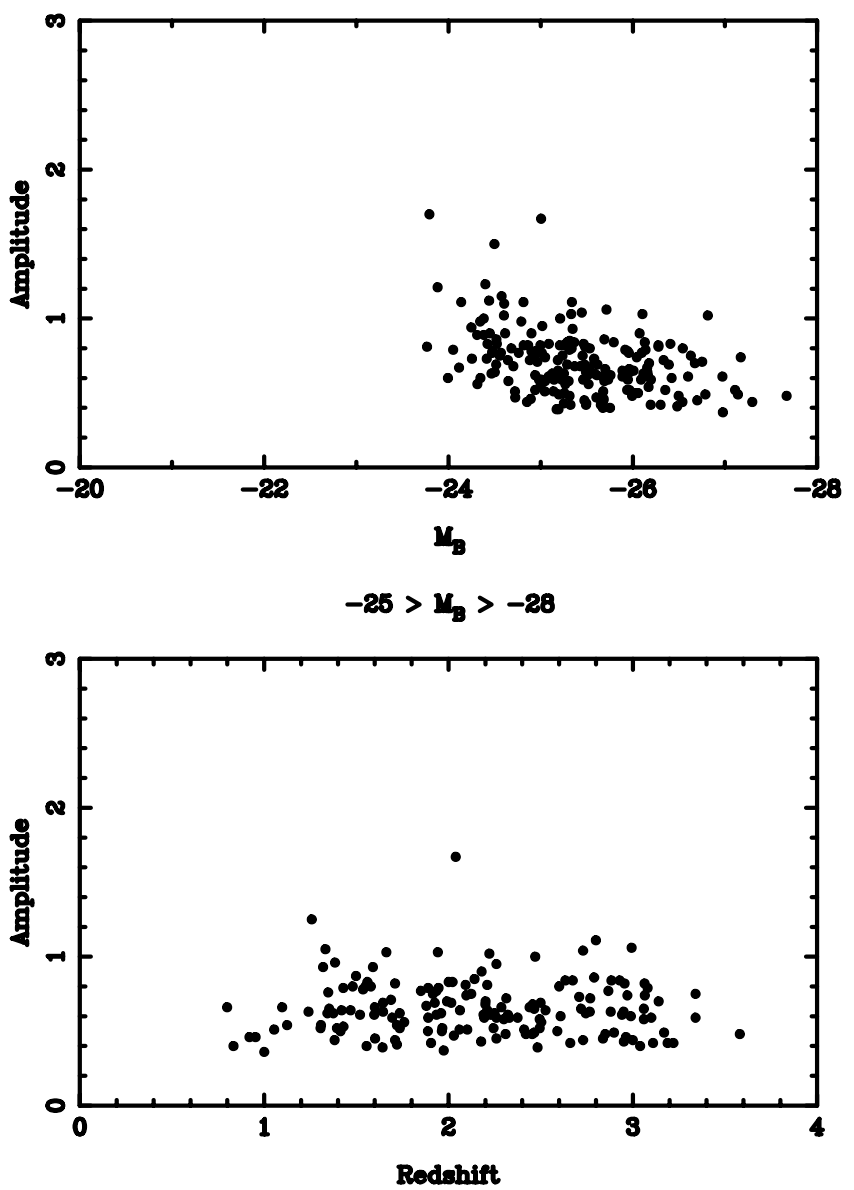

Fig. 5. Plots of amplitude versus redshift and absolute magnitude for the variability detected sample (VAR). The top two panels show amplitude versus absolute magnitude for two redshift ranges, and the bottom two panels show amplitude versus redshift for two absolute magnitude ranges

dependence. The net result in an un-binned sample cancels out with the luminosity variability relationship. Cid Fernandes et al.'s claim for a positive correlation between amplitude and redshift appears to be motivated at least in part by expectations arising from a paper by Di Clemente et al. (1996). This interesting paper examines the relation between their variability parameter $S_{1}$ (an amplitude based on the structure function) and wavelength. Their sample is composed of PG quasars (Schmidt \& Green 1983), which are mostly low redshift and relatively low luminosity objects. With the help of archival IUE observations they find that $S_{1}$ decreases with wavelength. This effect can clearly be seen in the light curve from the intensive monitoring programme of the Seyfert galaxy NGC 5548 (Clavel et al. 1991), which has a larger amplitude at shorter wavelength. Figure 8 shows the relation between amplitude and wavelength taken from the light curves, with a best-fit quadratic curve.

The relation between rest wavelength and amplitude is essentially equivalent to the relation between redshift and amplitude, where one is seeing progressively shorter wavelengths at higher redshift. This is illustrated in the top panel of Fig. 9 which shows the UVX sample with two curves superimposed. The solid line is converted from Fig. 8 and does not appear to follow the trend of the data, but the large scatter makes it hard to construct a convincing test. Nonetheless it suggests that any relation which holds for Seyfert galaxies might have to be modified for quasars. The dotted line is from Di Clemente et al. (1996), and shows a very small effect, which does nonetheless follow the flat distribution of the data.

The decrease of amplitude for the smallest redshift and luminosity seen in the top two panels of Fig. 4 has 

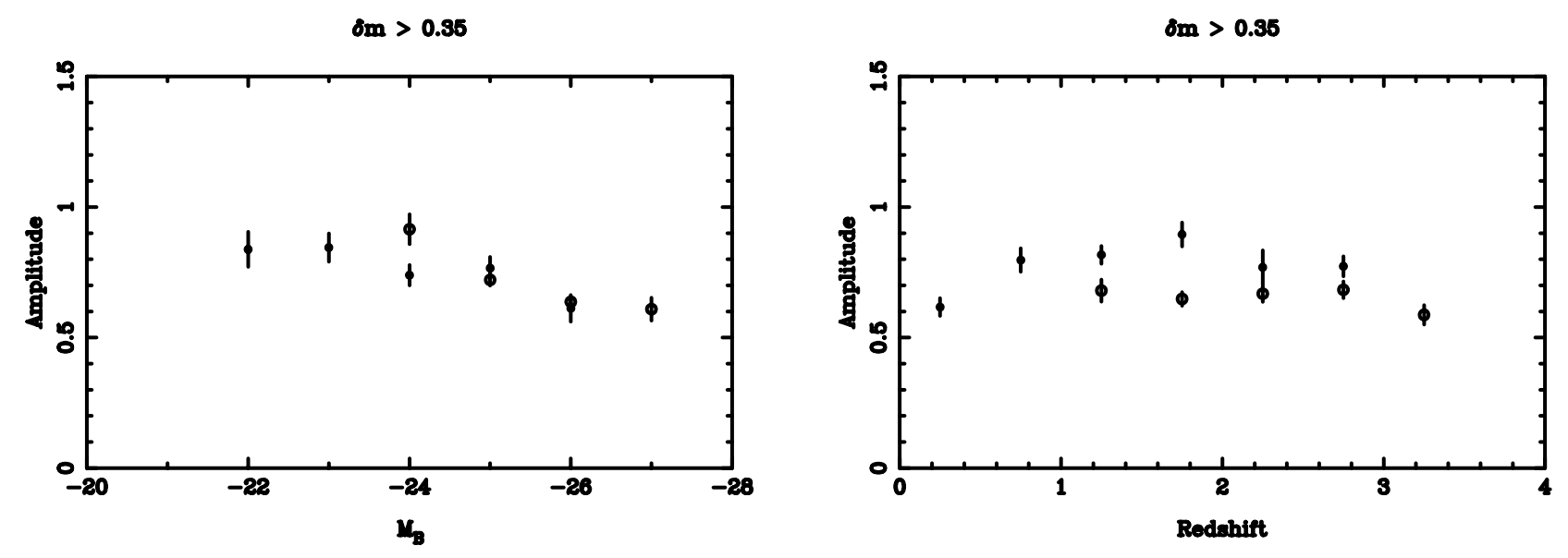

Fig. 6. Plots based on the same data as for Fig. 5, but binned in unit absolute magnitude intervals and redshift intervals of 0.5. The left hand panel shows the relation between amplitude and absolute magnitude for quasars with $z<1.5$ (small dots) and $z>1.5$ (large dots). The right hand panel shows the relation between amplitude and redshift for quasars with $M_{B}>-25$ (small dots) and $M_{B}<-25$ (large dots). The error bars show the uncertainty in the position of the mean

$\delta \mathrm{m}>1.1$

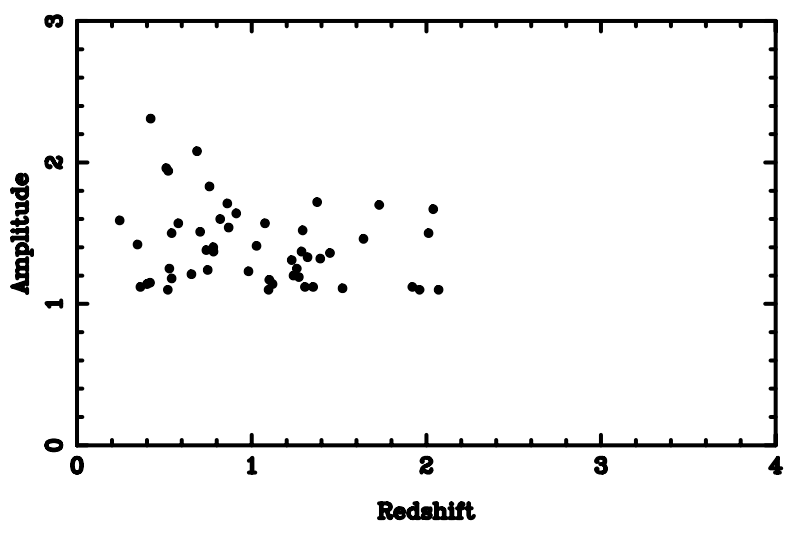

$\delta \mathrm{m}>1.1$

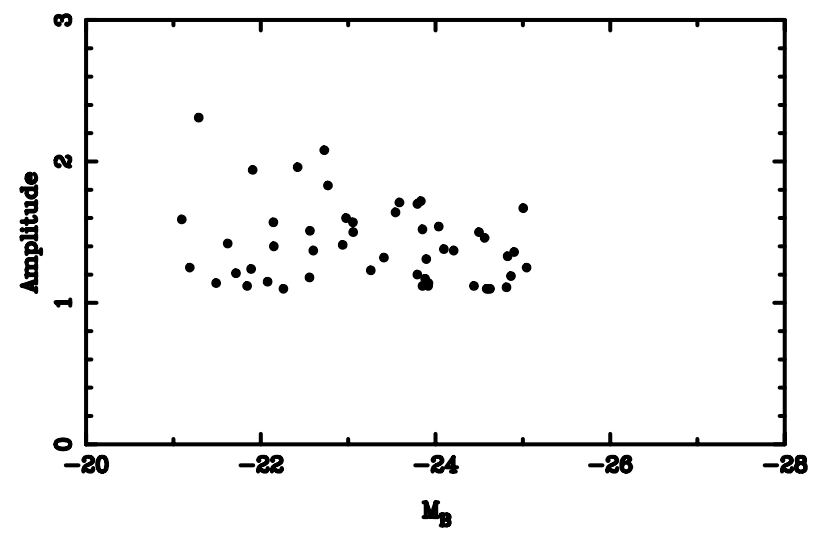

Fig. 7. Plots of amplitude versus redshift and absolute magnitude for the most variable quasars in the sample

a number of possible explanations. It could be the effect of the underlying galaxy dominating any change in nuclear brightness for low luminosity objects, it could be a consequence of the small optical depth to microlensing at low redshift, or it could be a consequence of the wavelength dependence of variability (Cristiani et al. 1996). The present dataset is not adequate to settle the question, which is best done by looking at luminous quasars at very low redshift and Seyfert galaxies at high redshift.

All the plots in Figs. 3 and 4 show a trend of decreasing amplitude towards higher redshift or more luminous objects. Although the trend as a function of luminosity is more marked, the old problem of degeneracy makes it hard to say for certain that it is a luminosity effect which is being observed. However, if we look at Fig. 6 where the data are binned in luminosity and redshift we see that while there is no significant trend of amplitude with redshift in either luminosity bin, there is a marked inverse correlation between amplitude and luminosity.

The relation between amplitude and luminosity is in agreement with that found in earlier work (Hook et al. 1994; Hawkins 1996; Cristiani et al. 1996) and may well turn out to be a useful way of distinguishing between various schemes for quasar variability. The evidence for a constant amplitude with redshift is more debatable. It would appear to be consistent with the early claim of Hook et al. (1994) for a weak anti-correlation with redshift which they ascribed to degeneracy with luminosity. It is also in 
agreement with the results of Cristiani et al. in the observer's frame. When they correct their structure function to the quasar rest frame they inevitably imprint a positive correlation between their variability parameter and redshift.

To investigate this dependence of amplitude on redshift for the present sample, Fig. 10 shows the epoch at which quasars achieve their maximum amplitude as a function of redshift. Apart from very low redshifts $(z<0.3)$ this relation is flat, implying that at least over the 21 years of the present dataset, time dilation effects will not bias the measurement of amplitude. Since the conclusions of Cid Fernandes et al. (1996) are largely based on a sample for which a time dilation correction has been applied, it is not feasible to make a direct comparison with the present work. However, it appears that the main difference between their results and those of Hook et al. is in the definition of a variability parameter and the method of analysis (both papers are based on the SGP sample).

There are perhaps three currently discussed schemes for quasar variability. The least well constrained is the accretion disk model, where instabilities are propagated across the disk leading to variation in light. The details of this approach have proved hard to work out, especially in the context of the constraints imposed by existing observations, but it does not seem to lead to an inverse correlation between amplitude and luminosity. An interesting recent attempt to model variation on the basis of accretion disk instabilities by Kawaguchi et al. (1998) may provide a means for producing the observed variations. It does however appear to predict variations which are either too asymmetric or of too small an amplitude to be consistent with the current observations. The timescales which they predict are also rather short, around 200 days for reasonable input parameters, and much shorter than the observed timescale of a few years.

An alternative approach, developed by Terlevich and his collaborators, accounts for the variation by postulating that the quasar is powered by a series of supernova explosions. Qualitatively, this model can account for the observed relation between luminosity and amplitude, and works quite well for Seyfert galaxies (Aretxaga \& Terlevich 1994). However, for quasars (Aretxaga et al. 1997) large numbers of supernovae are required to achieve the luminosity, which results in smaller variations. For example, even a relatively modest quasar with absolute magnitude $M_{B} \sim-26$ would need some 300 type II supernovae per year to power it, which given typical decay times would lead to very little variation at all. This clearly conflicts with observations in this paper which show that most quasars vary by around 0.5 to 1 magnitude on a timescale of a few years.

The third way of explaining quasar variability is to invoke microlensing. This approach has been explored in several recent papers (Hawkins 1993, 1996; Hawkins \& Taylor 1997), and seems to account well for a number of statistical properties of quasar light curves. It can also explain the inverse correlationi between luminosity and amplitude in a natural way. It is well known that when a point source is microlensed by a population of compact bodies of significant optical depth, the lenses combine nonlinearly to form a caustic pattern which produces sharp spikes in the resulting light curves (Schneider \& Weiss 1987). As the source becomes comparable in size with, or larger than, the Einstein radius of the lenses the amplitude decreases (Refsdal \& Stabell 1991). Thus if one assumes a uniform temperature for quasar disks, and that the luminosity is determined by the disk area, the larger more luminous disks will be amplified less. Using a relation between source size (in terms of Einstein radius) and amplitude given by Refsdal \& Stabell (1991) (Eq. 1), one can thus derive a relation between amplitude $\delta m$ and absolute magnitude $M$ of the form:

$\delta m=10^{0.2(M+c)}$

where $c$ is a constant. The bottom panel of Fig. 9 shows a plot of amplitude versus absolute magnitude for the UVX sample with this relation superimposed. The constant $c$ was adjusted to allow the curve to track the upper envelope of the points, which has the effect of defining the quasar disk size. This ranges from 1.1 Einstein radii for $M_{B}=-23$ to 11 for $M_{B}=-28$. The points scatter downwards from the upper envelope because the quasars do not necessarily attain their maximum possible amplitude. Although one cannot say that the curve provides a fit to the data, the trend is certainly well represented.

The possibility of a distinct population of large amplitude, low luminosity quasars suggested by Fig. 7 may also be used to test the models of quasar variability. Again, an accretion disk provides no obvious mechanism for such an effect. The Christmas Tree model certainly does imply large amplitude variations for low luminosity objects, where each event is of comparable brightness to the nucleus itself. Perhaps the most natural explanation comes from microlensing, where the nucleus of low luminosity quasars would plausibly become very small compared with the Einstein radii of the lenses, resulting in large amplifications from caustic crossing events.

\section{Conclusions}

In this paper we have examined the dependance of amplitude on redshift and luminosity for large samples of quasars selected on the basis of ultra-violet excess and variability. The quasars span a redshift range $0<z<3.5$ and a luminosity range $-20>M_{B}>-28$. There is evidence for a correlation of amplitude with luminosity and/or redshift for the sample as a whole, but when it is binned in redshift the correlation with luminosity becomes significant. This result could be strengthened by 


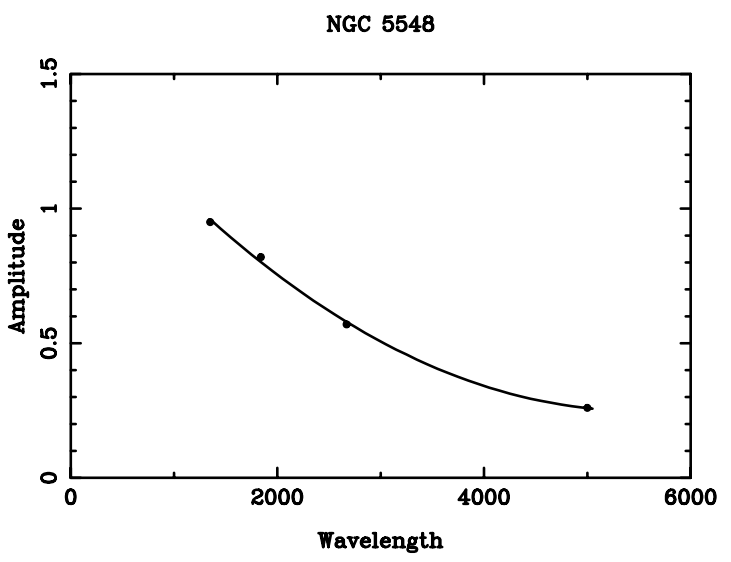

Fig. 8. Relation between amplitude and wavelength for the Seyfert galaxy NGC 5548 from IUE data published by Clavel et al. (1991)

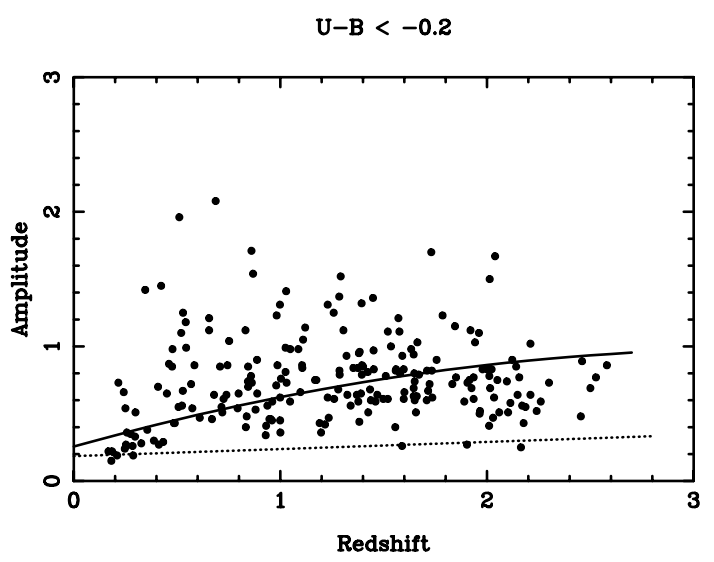

$\mathrm{U}-\mathrm{B}<-0.2$

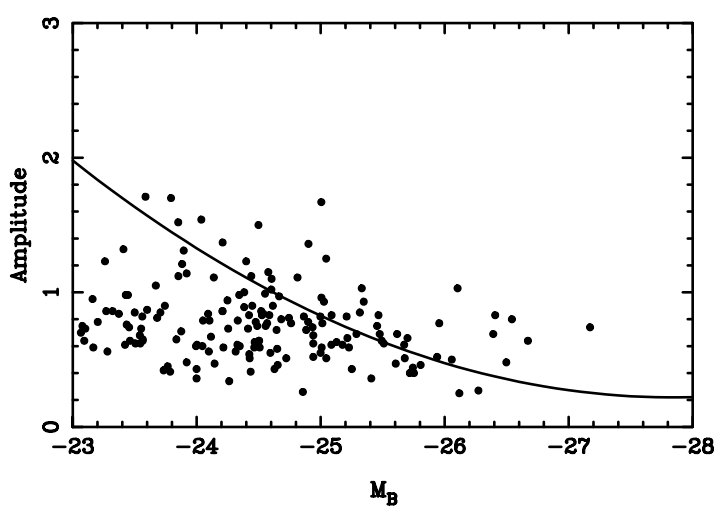

Fig. 9. Plots of amplitude versus redshift and luminosity for UVX selected quasars. In the top panel the solid line represents the relation from Fig. 8, with wavelength converted to redshift; the dotted line is the model from Di Clemente et al. (1996)

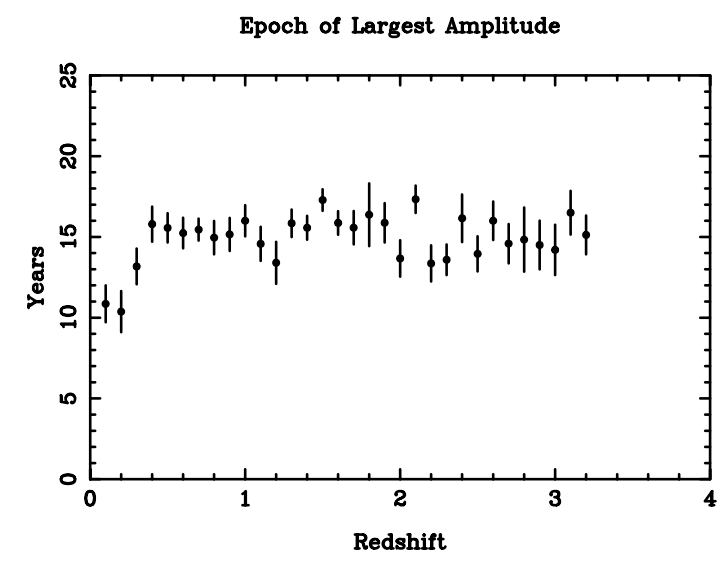

Fig. 10. Plot of the epoch at which the largest magnitude difference is achieved in quasar light curves at different redshifts. Poisson error bars are shown based on the number of objects in each bin

the possible existence of a population of large amplitude low luminosity objects in the sample. No convincing evidence is found for a correlation between amplitude and redshift, either for the sample as a whole or when it is binned in luminosity.

Various models of quasar variability are examined with respect to the observed correlations. It is concluded that any straightforward interpretation of an accretion disk model is incompatible with the data. The Christmas Tree model has some merits, especially in the regime of low luminosity quasars and Seyfert galaxies, but for luminous quasars the rate of supernovae required is too large to be compatible with the observed variability. The microlensing model can be used to explain all the data, although it does require that the Einstein radius of the microlensing bodies is comparable in size to the quasar nucleus.

Acknowledgements. I thank Andy Lawrence and Omar Almaini for making some excellent suggestions for improvements to the paper.

\section{References}

Aretxaga I., Terlevich R., 1994, MNRAS 269, 462

Aretxaga I., Cid Fernandes R., Terlevich R., 1997, MNRAS 286,271

Cid Fernandes R., Aretxaga I., Terlevich R., 1996, MNRAS 282,1191

Clavel J., et al., 1991, ApJ 366, 64

Cristiani S., Trentini S., La Franca F., Andreani P., 1996, A\&A 321,123

Cristiani S., Trentini S., La Franca F., Aretxaga I., Andreani P., Vio R., Gemmo A., 1996, A\&A 306, 395

Di Clemente A., Giallongo E., Natali G., Trèvese D., Vagnetti F., 1996, ApJ 463, 466 
Folkes S., et al., 1999, MNRAS 308, 459

Giallongo E., Trevese D., Vagnetti F., 1991, ApJ 377, 345

Hawkins M.R.S., 1993, Nat 366, 242

Hawkins M.R.S., 1996, MNRAS 278, 787

Hawkins M.R.S., Taylor A.N., 1997, ApJ 482, L5

Hawkins M.R.S., Véron P., 1995, MNRAS 275, 1102

Hook I.M., McMahon R.G., Boyle B.J., Irwin M.J., 1994, MNRAS 268, 305

Kawaguchi T., Mineshige S., Umemura M., Turner E.L., 1998, ApJ 504, 671
Morris S.L., Weymann R.J., Anderson S.F., Hewett P.C., Foltz C.B., Chaffee F.H., Francis P.J., MacAlpine G.M., 1991, AJ 102,1627

Refsdal S., Stabell R., 1991, A\&A 250, 62

Schmidt M., Green R.F., 1983, ApJ 269, 352

Schneider P., Weiss A., 1987, A\&A 171, 49

Trevese D., Pitella G., Kron R.G., Bershady M., 1989, AJ 98, 108

\section{Appendix}

\begin{tabular}{rrrrrrrrrrrrrr}
\multicolumn{2}{c}{ R.A. (1950) } & \multicolumn{2}{c}{ Dec $(1950)$} & $B$ & $U-B$ & $\delta m$ & $z$ & $M_{B}$ & Samples \\
21 & 16 & 28.38 & -44 & 0 & 57.3 & 19.34 & -0.42 & 0.64 & 2.062 & -26.04 & 1 & 0 & 0 \\
21 & 16 & 22.50 & -44 & 29 & 13.9 & 19.33 & -0.77 & 1.19 & 1.270 & -25.03 & 1 & 0 & 3 \\
21 & 16 & 44.32 & -44 & 9 & 28.0 & 20.29 & -0.59 & 1.72 & 1.375 & -24.24 & 0 & 0 & 3 \\
21 & 16 & 13.41 & -46 & 42 & 47.3 & 18.71 & -0.40 & 0.87 & 1.498 & -26.00 & 1 & 0 & 0 \\
21 & 16 & 25.78 & -46 & 10 & 42.1 & 18.92 & -0.37 & 0.54 & 0.748 & -24.32 & 1 & 0 & 0 \\
21 & 16 & 55.05 & -44 & 39 & 37.1 & 17.89 & -0.09 & 0.80 & 1.480 & -26.80 & 1 & 0 & 0 \\
21 & 17 & 24.47 & -42 & 41 & 4.1 & 19.51 & -0.35 & 1.12 & 0.363 & -22.17 & 0 & 0 & 3 \\
21 & 16 & 46.14 & -46 & 14 & 21.7 & 19.08 & -0.23 & 0.59 & 0.297 & -22.17 & 1 & 0 & 0 \\
21 & 17 & 30.01 & -44 & 2 & 40.4 & 18.30 & -0.45 & 0.44 & 1.710 & -26.69 & 1 & 0 & 0 \\
21 & 17 & 25.61 & -45 & 4 & 58.1 & 20.40 & 0.25 & 0.72 & 2.313 & -25.22 & 1 & 0 & 0 \\
21 & 17 & 26.97 & -45 & 30 & 20.9 & 20.28 & 1.15 & 1.06 & 2.993 & -25.86 & 1 & 0 & 0 \\
21 & 17 & 21.81 & -47 & 3 & 49.0 & 18.80 & 0.01 & 0.45 & 2.260 & -26.77 & 1 & 0 & 0 \\
21 & 17 & 53.12 & -46 & 0 & 44.8 & 19.71 & 0.31 & 0.82 & 2.955 & -26.41 & 1 & 0 & 0 \\
21 & 17 & 55.61 & -46 & 9 & 17.1 & 20.38 & 0.10 & 0.82 & 2.120 & -25.06 & 1 & 0 & 0 \\
21 & 17 & 48.23 & -46 & 47 & 36.2 & 19.35 & -0.50 & 0.48 & 1.038 & -24.59 & 1 & 0 & 0 \\
21 & 17 & 57.12 & -46 & 20 & 47.4 & 20.50 & 0.46 & 0.84 & 2.884 & -25.57 & 1 & 0 & 0 \\
21 & 18 & 21.07 & -44 & 59 & 51.9 & 19.19 & -0.12 & 0.59 & 2.194 & -26.32 & 1 & 0 & 0 \\
21 & 18 & 34.84 & -43 & 49 & 45.2 & 19.29 & -0.43 & 0.90 & 1.121 & -24.81 & 1 & 0 & 0 \\
21 & 18 & 34.30 & -44 & 0 & 44.9 & 20.44 & 1.01 & 0.70 & 3.140 & -25.80 & 1 & 0 & 0 \\
21 & 18 & 41.77 & -43 & 24 & 38.6 & 20.78 & -0.47 & 1.57 & 1.076 & -23.23 & 0 & 0 & 3
\end{tabular}




\begin{tabular}{|c|c|c|c|c|c|c|c|c|c|c|c|c|c|}
\hline \multicolumn{3}{|c|}{ R.A. (1950) } & \multicolumn{3}{|c|}{ Dec (1950) } & $B$ & $U-B$ & $\delta m$ & $z$ & $M_{B}$ & \multicolumn{3}{|c|}{ Samples } \\
\hline 21 & 18 & 47.48 & -43 & 1 & 9.5 & 18.81 & -0.62 & 0.75 & 2.123 & -26.63 & 1 & 0 & 0 \\
\hline 21 & 18 & 11.92 & -46 & 56 & 3.6 & 18.35 & -0.37 & 0.41 & 1.720 & -26.65 & 1 & 0 & 0 \\
\hline 21 & 18 & 55.69 & -43 & 3 & 36.1 & 18.66 & -0.01 & 0.70 & 2.201 & -26.85 & 1 & 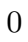 & 0 \\
\hline 21 & 19 & 0.54 & -43 & 48 & 0.5 & 20.72 & 0.63 & 0.82 & 3.064 & -25.47 & 1 & & 0 \\
\hline 21 & 18 & 29.22 & -47 & 2 & 34.9 & 18.36 & -0.42 & 1.05 & 1.332 & -26.10 & 1 & & 0 \\
\hline 21 & 19 & 14.89 & -43 & 7 & 27.0 & 19.81 & 0.43 & 0.84 & 2.929 & -26.29 & 1 & 0 & 0 \\
\hline 21 & 19 & 37.27 & -43 & 23 & 9.2 & 20.31 & 0.07 & 1.04 & 2.730 & -25.65 & 1 & 0 & 0 \\
\hline 21 & 19 & 20.05 & -45 & 30 & 52.3 & 20.38 & 0.00 & 0.51 & 2.410 & -25.32 & 1 & & 0 \\
\hline 21 & 19 & 45.46 & -43 & 32 & 19.3 & 20.65 & -0.65 & 1.40 & 0.652 & -22.29 & 0 & & 3 \\
\hline 21 & 19 & 47.61 & -43 & 46 & 23.9 & 19.16 & -0.40 & 1.02 & 1.000 & -24.70 & 1 & & 0 \\
\hline 21 & 19 & 37.16 & -45 & 48 & 22.3 & 19.98 & 0.03 & 0.81 & 2.210 & -25.54 & 1 & 0 & 0 \\
\hline 21 & 19 & 55.45 & -44 & 17 & 54.7 & 20.73 & 0.59 & 0.79 & 3.080 & -25.47 & 1 & & 0 \\
\hline 21 & 20 & 14.92 & -42 & 55 & 30.5 & 20.89 & 0.15 & 0.53 & 0.619 & -21.94 & 1 & & 0 \\
\hline 21 & 19 & 49.62 & -45 & 49 & 8.4 & 20.65 & -0.26 & 0.67 & 1.715 & -24.35 & 1 & 2 & 0 \\
\hline 21 & 20 & 18.19 & -44 & 10 & 40.6 & 20.69 & -0.10 & 1.10 & 0.580 & -22.00 & 0 & & 3 \\
\hline 21 & 20 & 28.49 & -43 & 38 & 36.6 & 19.23 & -0.35 & 0.51 & 0.840 & -24.26 & 1 & 0 & 0 \\
\hline 21 & 20 & 4.87 & -46 & 34 & 17.3 & 19.56 & 0.02 & 0.59 & 2.375 & -26.11 & 1 & & 0 \\
\hline 21 & 20 & 21.60 & -45 & 52 & 12.2 & 20.22 & 0.85 & 0.60 & 2.989 & -25.92 & 1 & & 0 \\
\hline 21 & 20 & 50.81 & -43 & 27 & 55.6 & 17.92 & -0.49 & 0.63 & 1.240 & -26.39 & 1 & & 0 \\
\hline 21 & 20 & 58.76 & -44 & 13 & 48.6 & 20.94 & 0.02 & 0.81 & 1.741 & -24.09 & 1 & 0 & 0 \\
\hline 21 & 20 & 47.86 & -45 & 32 & 44.2 & 20.03 & 1.10 & 0.61 & 2.941 & -26.08 & 1 & & 0 \\
\hline 21 & 20 & 57.48 & -46 & 20 & 36.2 & 20.09 & -0.35 & 0.64 & 0.739 & -23.12 & 1 & & 0 \\
\hline 21 & 21 & 26.81 & -43 & 51 & 34.2 & 19.20 & -0.49 & 0.79 & 1.946 & -26.06 & 1 & 0 & 0 \\
\hline 21 & 21 & 20.68 & -44 & 45 & 7.7 & 20.29 & 0.98 & 0.46 & 2.963 & -25.83 & 1 & & 0 \\
\hline 21 & 21 & 19.34 & -45 & 5 & 0.8 & 21.18 & -0.07 & 1.24 & 0.748 & -22.06 & 0 & & 3 \\
\hline 21 & 21 & 12.02 & -46 & 4 & 1.4 & 20.34 & 0.09 & 0.95 & 2.260 & -25.23 & 1 & & 0 \\
\hline 21 & 21 & 22.69 & -45 & 58 & 25.6 & 20.74 & -0.32 & 0.60 & 1.650 & -24.17 & 0 & & 0 \\
\hline 21 & 21 & 41.90 & -44 & 6 & 57.6 & 17.80 & -0.46 & 0.52 & 1.735 & -27.22 & 1 & 0 & 0 \\
\hline 21 & 21 & 33.83 & -45 & 15 & 9.2 & 20.39 & -0.11 & 1.83 & 0.758 & -22.88 & 0 & 0 & 3 \\
\hline 21 & 21 & 23.86 & -46 & 38 & 7.0 & 19.92 & -0.21 & 1.64 & 0.912 & -23.74 & 0 & & 3 \\
\hline 21 & 21 & 31.37 & -46 & 13 & 36.3 & 20.51 & -0.48 & 0.59 & 1.047 & -23.45 & 0 & & 0 \\
\hline 21 & 21 & 43.13 & -44 & 56 & 36.3 & 20.40 & 0.38 & 0.63 & 2.950 & -25.71 & 1 & 0 & 0 \\
\hline 21 & 21 & 40.68 & -45 & 51 & 23.9 & 18.89 & -0.43 & 0.46 & 0.947 & -24.85 & 1 & 2 & 0 \\
\hline 21 & 21 & 42.39 & -45 & 49 & 26.6 & 20.10 & -0.28 & 1.10 & 0.520 & -22.36 & 1 & 2 & 3 \\
\hline 21 & 21 & 35.46 & -46 & 42 & 27.4 & 19.11 & -0.41 & 0.76 & 1.347 & -25.38 & 1 & & 0 \\
\hline 21 & 21 & 41.39 & -45 & 59 & 53.9 & 19.73 & -0.58 & 0.90 & 0.887 & -23.87 & 1 & 2 & 0 \\
\hline 21 & 21 & 39.65 & -46 & 41 & 17.2 & 20.35 & -0.52 & 1.12 & 1.352 & -24.15 & 0 & 0 & 3 \\
\hline 21 & 21 & 56.68 & -45 & 8 & 33.6 & 18.83 & -0.31 & 0.65 & 1.353 & -25.67 & 1 & 0 & 0 \\
\hline 21 & 22 & 12.50 & -43 & 9 & 36.5 & 20.83 & 0.46 & 0.65 & 3.060 & -25.36 & 1 & & 0 \\
\hline 21 & 22 & 0.52 & -45 & 57 & 24.8 & 17.75 & -0.42 & 0.46 & 0.953 & -26.01 & 0 & 2 & 0 \\
\hline 21 & 22 & 25.85 & -42 & 43 & 24.2 & 20.16 & 0.17 & 0.68 & 2.457 & -25.58 & 1 & 0 & 0 \\
\hline 21 & 22 & 30.46 & -43 & 28 & 38.6 & 20.01 & -0.10 & 1.14 & 0.401 & -21.89 & 0 & 0 & 3 \\
\hline 21 & 22 & 25.60 & -44 & 22 & 18.5 & 18.87 & 0.22 & 0.49 & 2.465 & -26.88 & 1 & 0 & 0 \\
\hline 21 & 22 & 16.68 & -46 & 31 & 54.8 & 20.27 & -0.10 & 1.60 & 0.820 & -23.17 & 0 & 0 & 3 \\
\hline 21 & 22 & 39.06 & -44 & 33 & 4.8 & 20.32 & 0.05 & 0.80 & 2.600 & -25.54 & 1 & 0 & 0 \\
\hline 21 & 22 & 41.46 & -44 & 47 & 46.1 & 19.92 & 0.06 & 1.05 & 1.137 & -24.21 & 1 & 0 & 0 \\
\hline 21 & 22 & 52.08 & -43 & 33 & 58.6 & 18.71 & -0.07 & 0.45 & 0.552 & -23.88 & 1 & 0 & 0 \\
\hline 21 & 22 & 35.59 & -46 & 22 & 5.7 & 19.06 & -0.37 & 0.81 & 2.093 & -26.35 & 1 & 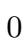 & 0 \\
\hline 21 & 22 & 48.18 & -45 & 55 & 30.6 & 19.96 & -0.53 & 0.51 & 1.425 & -24.65 & 1 & 2 & 0 \\
\hline 21 & 22 & 49.75 & -45 & 58 & 24.4 & 19.41 & -0.31 & 0.86 & 0.986 & -24.42 & 1 & 2 & 0 \\
\hline 21 & 22 & 56.31 & -45 & 57 & 17.2 & 20.13 & -0.28 & 0.78 & 0.858 & -23.40 & 1 & 2 & 0 \\
\hline 21 & 23 & 1.03 & -46 & 3 & 38.1 & 19.42 & -0.61 & 0.96 & 1.384 & -25.13 & 1 & 2 & 0 \\
\hline 21 & 23 & 3.18 & -46 & 5 & 48.0 & 20.53 & 0.70 & 0.42 & 3.220 & -25.76 & 1 & 0 & 0 \\
\hline 21 & 23 & 21.19 & -43 & 38 & 29.5 & 18.37 & -0.18 & 0.66 & 0.480 & -23.92 & 1 & 0 & 0 \\
\hline 21 & 23 & 3.71 & -46 & 54 & 52.7 & 18.63 & -0.24 & 0.79 & 1.429 & -25.98 & 1 & 0 & 0 \\
\hline
\end{tabular}




\begin{tabular}{|c|c|c|c|c|c|c|c|c|c|c|c|c|c|}
\hline \multicolumn{3}{|c|}{ R.A. (1950) } & \multicolumn{3}{|c|}{ Dec (1950) } & \multirow{2}{*}{$\begin{array}{c}B \\
19.12\end{array}$} & \multirow{2}{*}{$\begin{array}{l}U-B \\
-0.22\end{array}$} & \multirow{2}{*}{$\begin{array}{c}\delta m \\
0.80\end{array}$} & \multirow{2}{*}{$\begin{array}{c}z \\
1.549\end{array}$} & \multirow{2}{*}{$\begin{array}{c}M_{B} \\
-25.66\end{array}$} & \multicolumn{3}{|c|}{ Samples } \\
\hline 21 & 23 & 14.08 & -45 & 48 & 53.9 & & & & & & 1 & 0 & 0 \\
\hline 21 & 23 & 31.47 & -42 & 56 & 35.7 & 19.49 & 0.23 & 1.26 & 0.141 & -20.15 & 1 & 0 & 3 \\
\hline 21 & 23 & 13.92 & -46 & 54 & 33.0 & 20.34 & -0.10 & 2.31 & 0.422 & -21.67 & 0 & & 3 \\
\hline 21 & 23 & 37.93 & -43 & 46 & 58.0 & 18.91 & -0.44 & 0.58 & 1.000 & -24.95 & 1 & & 0 \\
\hline 21 & 23 & 43.22 & -44 & 29 & 5.9 & 19.66 & -0.66 & 0.84 & 1.353 & -24.84 & 1 & & 0 \\
\hline 21 & 23 & 45.16 & -44 & 29 & 9.8 & 20.06 & -0.08 & 1.17 & 1.586 & -24.77 & 0 & & 3 \\
\hline 21 & 23 & 48.33 & -45 & 38 & 4.0 & 19.50 & -0.28 & 0.39 & 1.642 & -25.40 & 1 & & 0 \\
\hline 21 & 24 & 7.74 & -44 & 12 & 53.9 & 19.39 & -0.16 & 1.50 & 0.542 & -23.16 & 1 & & 3 \\
\hline 21 & 24 & 5.49 & -44 & 43 & 57.5 & 19.83 & -0.32 & 0.26 & 1.589 & -25.01 & 0 & & 0 \\
\hline 21 & 24 & 9.52 & -44 & 12 & 58.3 & 20.26 & -0.43 & 1.20 & 1.239 & -24.05 & 0 & & 3 \\
\hline 21 & 24 & 3.30 & -46 & 27 & 37.3 & 20.78 & 0.00 & 0.52 & 1.140 & -23.36 & 1 & & 0 \\
\hline 21 & 24 & 7.41 & -46 & 5 & 44.6 & 20.45 & 0.06 & 1.51 & 0.705 & -22.66 & 1 & & 3 \\
\hline 21 & 24 & 11.89 & -45 & 40 & 17.0 & 18.78 & -0.52 & 0.52 & 1.395 & -25.78 & 1 & & 0 \\
\hline 21 & 24 & 19.46 & -45 & 15 & 1.7 & 20.03 & -0.51 & 0.61 & 1.935 & -25.22 & 1 & & 0 \\
\hline 21 & 24 & 30.39 & -43 & 3 & 34.7 & 19.46 & -0.39 & 0.54 & 1.286 & -24.93 & 1 & & 0 \\
\hline 21 & 24 & 33.74 & -44 & 12 & 3.5 & 20.01 & -0.34 & 1.88 & 1.625 & -24.87 & 0 & & 3 \\
\hline 21 & 24 & 21.85 & -46 & 52 & 0.4 & 19.90 & 0.58 & 0.58 & 2.495 & -25.87 & 1 & & 0 \\
\hline 21 & 24 & 49.75 & -43 & 20 & 36.0 & 19.01 & 0.07 & 0.64 & 0.820 & -24.43 & 1 & & 0 \\
\hline 21 & 24 & 46.25 & -44 & 32 & 24.7 & 20.66 & 0.16 & 0.47 & 0.677 & -22.37 & 1 & & 0 \\
\hline 21 & 24 & 46.53 & -45 & 53 & 46.9 & 20.48 & -0.62 & 1.10 & 1.961 & -24.79 & 0 & & 3 \\
\hline 21 & 24 & 54.69 & -45 & 4 & 54.4 & 20.48 & -0.32 & 0.30 & 0.388 & -21.35 & 0 & & 0 \\
\hline 21 & 24 & 56.51 & -45 & 53 & 2.6 & 19.76 & 0.03 & 0.61 & 2.322 & -25.86 & 1 & & 0 \\
\hline 21 & 25 & 5.28 & -44 & 48 & 42.3 & 19.91 & -0.40 & 0.82 & 1.733 & -25.11 & 0 & & 0 \\
\hline 21 & 25 & 25.85 & -43 & 35 & 13.5 & 20.32 & 0.01 & 0.59 & 2.260 & -25.25 & 1 & & 0 \\
\hline 21 & 25 & 23.50 & -44 & 24 & 24.2 & 20.01 & -0.16 & 0.69 & 2.500 & -25.77 & 1 & & 0 \\
\hline 21 & 25 & 23.30 & -45 & 16 & 17.9 & 20.62 & -0.28 & 0.66 & 0.242 & -20.19 & 1 & & 0 \\
\hline 21 & 25 & 28.96 & -45 & 9 & 50.0 & 19.41 & -0.38 & 0.93 & 1.590 & -25.43 & 1 & & 0 \\
\hline 21 & 25 & 28.12 & -45 & 36 & 53.9 & 20.44 & 0.24 & 0.63 & 2.766 & -25.54 & 1 & U & 0 \\
\hline 21 & 25 & 28.84 & -46 & 39 & 17.1 & 19.11 & -0.41 & 0.45 & 1.601 & -25.74 & 1 & & 0 \\
\hline 21 & 25 & 46.60 & -44 & 32 & 58.3 & 20.39 & -0.04 & 0.56 & 2.503 & -25.39 & 1 & & 0 \\
\hline 21 & 25 & 53.43 & -43 & 43 & 39.9 & 20.44 & -0.29 & 0.65 & 0.451 & -21.71 & 1 & & 0 \\
\hline 21 & 25 & 56.64 & -44 & 8 & 32.1 & 18.48 & -0.33 & 0.63 & 0.366 & -23.22 & 1 & 0 & 0 \\
\hline 21 & 25 & 49.78 & -46 & 11 & 32.1 & 20.32 & -0.78 & 1.12 & 1.305 & -24.10 & 1 & & 3 \\
\hline 21 & 25 & 49.84 & -46 & 47 & 52.4 & 19.07 & -0.45 & 0.50 & 1.888 & -26.13 & 1 & & 0 \\
\hline 21 & 26 & 10.02 & -42 & 56 & 30.8 & 17.79 & -0.28 & 0.67 & 0.405 & -24.13 & 1 & & 0 \\
\hline 21 & 26 & 1.19 & -47 & 8 & 26.6 & 19.28 & -0.10 & 0.52 & 0.698 & -23.81 & 1 & 0 & 0 \\
\hline 21 & 26 & 7.35 & -45 & 34 & 52.2 & 21.27 & -0.22 & 0.51 & 0.719 & -21.88 & 0 & 2 & 0 \\
\hline 21 & 26 & 12.68 & -47 & 8 & 30.4 & 20.04 & 0.02 & 0.68 & 2.200 & -25.47 & 1 & & 0 \\
\hline 21 & 26 & 33.72 & -45 & 58 & 45.3 & 18.17 & -0.23 & 0.80 & 1.579 & -26.65 & 1 & & 0 \\
\hline 21 & 26 & 40.88 & -43 & 34 & 19.4 & 18.48 & -0.27 & 0.86 & 0.584 & -24.23 & 1 & & 0 \\
\hline 21 & 26 & 43.20 & -43 & 36 & 13.8 & 20.71 & -0.49 & 0.68 & 1.280 & -23.67 & 1 & 2 & 0 \\
\hline 21 & 26 & 39.70 & -45 & 2 & 43.9 & 20.54 & -0.49 & 0.77 & 2.156 & -24.93 & 0 & 2 & 0 \\
\hline 21 & 26 & 41.19 & -45 & 31 & 12.6 & 19.93 & -0.56 & 0.71 & 0.980 & -23.89 & 1 & & 0 \\
\hline 21 & 26 & 45.68 & -43 & 50 & 6.3 & 20.08 & -0.37 & 1.14 & 1.1 & -24.02 & 1 & & 3 \\
\hline 21 & 26 & 50.42 & -43 & 46 & 50.6 & 20.29 & -0.47 & 1.05 & 1.110 & -23.79 & 1 & 2 & 0 \\
\hline 21 & 26 & 49.67 & -45 & 26 & 56.3 & 18.59 & -0.47 & 0.44 & 1.382 & -25.95 & 1 & 2 & 0 \\
\hline 21 & 26 & 52.97 & -46 & 19 & 0.7 & 18.90 & -0.66 & 0.67 & 1.880 & -26.29 & 1 & 0 & 0 \\
\hline 21 & 27 & 0.30 & -44 & 56 & 34.0 & 20.33 & -0.16 & 1.94 & 0.522 & -22.14 & 0 & & 3 \\
\hline 21 & 26 & 58.26 & -47 & 2 & 58.9 & 20.69 & 0.08 & 0.90 & 2.592 & -25.16 & 1 & 0 & 0 \\
\hline 21 & 27 & 8.64 & -44 & 29 & 24.6 & 20.83 & -0.20 & 0.90 & 2.122 & -24.61 & 0 & 2 & 0 \\
\hline 21 & 27 & 13.56 & -44 & 35 & 17.7 & 18.82 & -0.57 & 0.69 & 2.015 & -26.51 & 1 & 2 & 0 \\
\hline 21 & 27 & 19.33 & -42 & 42 & 44.3 & 17.71 & -0.41 & 0.66 & 0.799 & -25.67 & 1 & U & 0 \\
\hline 21 & 27 & 14.13 & -45 & 55 & 19.9 & 20.12 & 0.15 & 0.66 & 2.440 & -25.61 & 1 & & 0 \\
\hline 21 & 27 & 19.62 & -43 & 45 & 11.4 & 20.08 & -0.46 & 0.72 & 1.722 & -24.92 & 1 & 2 & 0 \\
\hline 21 & 27 & 17.65 & -46 & 1 & 39.1 & 19.98 & -0.94 & 1.37 & 1.285 & -24.41 & 1 & 2 & 3 \\
\hline
\end{tabular}




\begin{tabular}{|c|c|c|c|c|c|c|c|c|c|c|c|c|c|}
\hline \multicolumn{3}{|c|}{ R.A. (1950) } & \multicolumn{3}{|c|}{ Dec (1950) } & \multirow{2}{*}{$\begin{array}{c}B \\
19.83\end{array}$} & \multirow{2}{*}{$\begin{array}{r}U-B \\
0.43\end{array}$} & \multirow{2}{*}{$\begin{array}{l}\delta m \\
0.63\end{array}$} & \multirow{2}{*}{$\begin{array}{c}z \\
2.880\end{array}$} & \multirow{2}{*}{$\begin{array}{c}M_{B} \\
-26.24\end{array}$} & \multicolumn{3}{|c|}{ Samples } \\
\hline 21 & 27 & 21.35 & -44 & 56 & 24.6 & & & & & & 1 & 0 & 0 \\
\hline 21 & 27 & 31.32 & -44 & 45 & 6.4 & 18.72 & -0.35 & 0.78 & 1.536 & -26.04 & 1 & 0 & 0 \\
\hline 21 & 27 & 39.34 & -43 & 8 & 36.7 & 20.46 & 0.00 & 0.84 & 2.674 & -25.45 & 1 & & 0 \\
\hline 21 & 27 & 37.22 & -45 & 28 & 57.0 & 18.60 & 0.84 & 0.44 & 2.730 & -27.36 & 1 & & 0 \\
\hline 21 & 27 & 52.99 & -43 & 50 & 37.0 & 19.40 & -0.15 & 0.64 & 0.493 & -22.94 & 1 & & 0 \\
\hline 21 & 27 & 54.41 & -45 & 52 & 55.5 & 19.94 & -0.48 & 1.17 & 1.101 & -24.12 & 0 & & 3 \\
\hline 21 & 27 & 55.93 & -45 & 49 & 0.5 & 19.04 & -0.15 & 1.07 & 0.871 & -24.52 & 1 & & 0 \\
\hline 21 & 27 & 55.24 & -47 & 6 & 50.1 & 18.42 & -0.24 & 0.57 & 0.578 & -24.27 & 1 & & 0 \\
\hline 21 & 27 & 59.09 & -45 & 11 & 9.3 & 19.83 & -0.89 & 0.83 & 2.001 & -25.49 & 1 & & 0 \\
\hline 21 & 28 & 2.17 & -45 & 25 & 10.3 & 19.01 & -0.37 & 0.59 & 0.961 & -24.76 & 0 & & 0 \\
\hline 21 & 28 & 4.28 & -46 & 39 & 7.9 & 20.73 & 0.05 & 0.71 & 2.610 & -25.13 & 1 & & 0 \\
\hline 21 & 28 & 5.82 & -46 & 33 & 24.7 & 17.44 & 0.19 & 0.65 & 0.153 & -22.37 & 1 & & 0 \\
\hline 21 & 28 & 14.96 & -42 & 45 & 41.9 & 19.44 & -0.38 & 0.64 & 1.420 & -25.16 & 1 & & 0 \\
\hline 21 & 28 & 13.84 & -44 & 43 & 32.1 & 20.49 & -0.23 & 0.62 & 1.229 & -23.81 & 1 & & 0 \\
\hline 21 & 28 & 17.05 & -44 & 34 & 56.0 & 19.52 & -0.53 & 0.68 & 1.434 & -25.10 & 1 & & 0 \\
\hline 21 & 28 & 21.96 & -46 & 10 & 53.2 & 17.65 & -0.50 & 0.40 & 0.833 & -25.82 & 1 & & 0 \\
\hline 21 & 28 & 24.69 & -45 & 37 & 3.4 & 19.77 & 0.00 & 0.70 & 0.204 & -20.67 & 1 & & 0 \\
\hline 21 & 28 & 40.98 & -45 & 20 & 20.8 & 20.56 & -0.25 & 1.75 & 0.544 & -22.00 & 0 & & 3 \\
\hline 21 & 28 & 42.00 & -43 & 30 & 59.2 & 18.91 & -0.60 & 0.42 & 1.905 & -26.30 & 1 & & 0 \\
\hline 21 & 28 & 44.77 & -46 & 4 & 16.3 & 20.51 & -0.20 & 1.12 & 0.831 & -22.95 & 1 & & 0 \\
\hline 21 & 28 & 56.74 & -45 & 48 & 1.9 & 20.45 & -0.42 & 0.98 & 1.087 & -23.59 & 0 & & 0 \\
\hline 21 & 29 & 1.26 & -46 & 6 & 30.4 & 20.07 & -0.40 & 0.81 & 1.025 & -23.84 & 1 & & 0 \\
\hline 21 & 29 & 4.08 & -42 & 55 & 55.0 & 20.65 & -0.46 & 1.10 & 2.070 & -24.74 & 0 & & 3 \\
\hline 21 & 29 & 4.72 & -44 & 42 & 39.7 & 19.22 & -0.38 & 0.99 & 1.025 & -24.69 & 1 & & 0 \\
\hline 21 & 29 & 8.42 & -45 & 15 & 39.0 & 19.80 & -0.67 & 0.61 & 1.260 & -24.55 & 1 & & 0 \\
\hline 21 & 29 & 10.18 & -46 & 17 & 2.3 & 20.74 & -0.30 & 1.31 & 0.998 & -23.11 & 1 & & 0 \\
\hline 21 & 29 & 10.53 & -44 & 50 & 18.5 & 19.07 & -0.68 & 1.03 & 1.942 & -26.18 & 1 & & 0 \\
\hline 21 & 29 & 24.73 & -45 & 27 & 7.0 & 20.21 & -0.66 & 0.84 & 1.375 & -24.32 & 1 & 2 & 0 \\
\hline 21 & 29 & 27.63 & -45 & 1 & 15.8 & 20.89 & -0.08 & 0.56 & 2.170 & -24.59 & 1 & & 0 \\
\hline 21 & 29 & 32.92 & -45 & 51 & 30.9 & 20.58 & -0.35 & 0.82 & 1.286 & -23.81 & 1 & & 0 \\
\hline 21 & 29 & 38.02 & -45 & 12 & 11.6 & 19.31 & 0.11 & 0.90 & 2.180 & -26.18 & 1 & & 0 \\
\hline 21 & 29 & 38.26 & -44 & 13 & 47.1 & 19.85 & -0.45 & 0.83 & 1.452 & -24.80 & 1 & 2 & 0 \\
\hline 21 & 29 & 39.50 & -46 & 24 & 19.2 & 17.80 & -0.30 & 0.67 & 0.435 & -24.27 & 1 & & 0 \\
\hline 21 & 29 & 39.63 & -46 & 29 & 40.3 & 19.71 & 0.02 & 0.65 & 2.465 & -26.04 & 1 & & 0 \\
\hline 21 & 29 & 41.25 & -45 & 22 & 49.0 & 20.21 & 9.99 & 0.48 & 3.580 & -26.30 & 1 & & 0 \\
\hline 21 & 29 & 42.67 & -45 & 16 & 27.2 & 20.62 & -0.54 & 0.73 & 1.030 & -23.30 & 1 & 2 & 0 \\
\hline 21 & 29 & 45.45 & -46 & 53 & 47.6 & 18.85 & -0.02 & 0.61 & 2.230 & -26.69 & 1 & 0 & 0 \\
\hline 21 & 29 & 47.37 & -46 & 2 & 7.8 & 18.97 & -0.27 & 0.51 & 0.299 & -22.29 & 1 & & 0 \\
\hline 21 & 29 & 50.05 & -45 & 46 & 56.6 & 20.29 & -0.25 & 0.62 & 2.034 & -25.06 & 0 & & 0 \\
\hline 21 & 29 & 51.34 & -44 & 25 & 9.8 & 20.71 & -0.19 & 0.86 & 0.744 & -22.52 & 1 & U & 0 \\
\hline 21 & 29 & 57.55 & -46 & 53 & 51.1 & 19.85 & 0.02 & 0.62 & 2.208 & -25.67 & 1 & 0 & 0 \\
\hline 21 & 29 & 56.13 & -44 & 23 & 10.5 & 20.76 & -0.67 & 0.78 & 2.011 & -24.57 & 0 & 2 & 0 \\
\hline 21 & 30 & 1.27 & -46 & 3 & 4.9 & 19.61 & -0.14 & 0.52 & 2.244 & -25.94 & 1 & & 0 \\
\hline 21 & 30 & 1.99 & -44 & 3 & 36.7 & 20.88 & 0.68 & 0.74 & & -25.25 & 1 & & 0 \\
\hline 21 & 30 & 2.98 & -45 & 8 & 45.2 & 19.07 & 0.05 & 1.38 & 0.740 & -24.15 & 1 & 0 & 3 \\
\hline 21 & 30 & 8.22 & -43 & 52 & 59.0 & 19.93 & 0.11 & 0.84 & 2.634 & -25.95 & 1 & 0 & 0 \\
\hline 21 & 30 & 16.27 & -43 & 10 & 28.9 & 21.37 & -0.16 & 1.41 & 0.914 & -22.30 & 0 & 0 & 3 \\
\hline 21 & 30 & 20.02 & -44 & 40 & 42.8 & 20.25 & -0.07 & 0.47 & 0.610 & -22.55 & 1 & & 0 \\
\hline 21 & 30 & 20.78 & -44 & 45 & 36.3 & 20.00 & -0.48 & 0.59 & 1.460 & -24.66 & 0 & & 0 \\
\hline 21 & 30 & 22.37 & -44 & 50 & 58.9 & 19.65 & -0.12 & 0.61 & 0.725 & -23.52 & 1 & 0 & 0 \\
\hline 21 & 30 & 24.58 & -44 & 42 & 19.8 & 20.29 & 0.96 & 0.40 & 3.040 & -25.89 & 1 & 0 & 0 \\
\hline 21 & 30 & 27.93 & -45 & 55 & 32.9 & 18.91 & -0.31 & 0.40 & 1.556 & -25.88 & 1 & 2 & 0 \\
\hline 21 & 30 & 30.00 & -45 & 45 & 38.2 & 20.65 & 0.23 & 0.59 & 2.645 & -25.24 & 1 & & 0 \\
\hline 21 & 30 & 30.16 & -44 & 45 & 24.4 & 19.71 & -0.63 & 0.64 & 1.324 & -24.74 & 0 & 2 & 0 \\
\hline 21 & 30 & 30.63 & -43 & 6 & 51.6 & 19.51 & -0.23 & 0.69 & 1.645 & -25.40 & 1 & 2 & 0 \\
\hline
\end{tabular}




\begin{tabular}{|c|c|c|c|c|c|c|c|c|c|c|c|c|c|}
\hline \multicolumn{3}{|c|}{ R.A. (1950) } & \multicolumn{3}{|c|}{ Dec (1950) } & \multirow{2}{*}{$\begin{array}{c}B \\
20.22\end{array}$} & \multirow{2}{*}{$\begin{array}{l}U-B \\
-0.69\end{array}$} & \multirow{2}{*}{$\begin{array}{l}\delta m \\
1.31\end{array}$} & \multirow{2}{*}{$\begin{array}{c}z \\
1.229\end{array}$} & \multirow{2}{*}{$\begin{array}{c}M_{B} \\
-24.08\end{array}$} & \multicolumn{3}{|c|}{ Samples } \\
\hline 21 & 30 & 38.92 & -43 & 50 & 22.4 & & & & & & 1 & 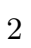 & 3 \\
\hline 21 & 30 & 41.40 & -43 & 48 & 1.0 & 19.56 & -0.40 & 0.61 & 1.597 & -25.29 & 1 & & 0 \\
\hline 21 & 30 & 45.86 & -45 & 56 & 38.6 & 20.58 & -0.26 & 0.61 & 1.498 & -24.13 & 1 & & 0 \\
\hline 21 & 30 & 50.85 & -44 & 32 & 7.2 & 18.85 & -0.27 & 0.54 & 0.793 & -24.51 & 1 & & 0 \\
\hline 21 & 31 & 1.26 & -46 & 7 & 4.0 & 20.73 & -0.39 & 0.41 & 2.010 & -24.60 & 0 & & 0 \\
\hline 21 & 31 & 0.28 & -44 & 36 & 23.0 & 19.99 & -0.36 & 0.47 & 1.234 & -24.31 & 1 & & 0 \\
\hline 21 & 31 & 7.60 & -46 & 12 & 42.8 & 20.71 & -0.40 & 0.83 & 1.978 & -24.58 & 0 & & 0 \\
\hline 21 & 31 & 4.84 & -44 & 14 & 6.4 & 20.23 & -0.44 & 0.62 & 0.999 & -23.63 & 0 & & 0 \\
\hline 21 & 31 & 12.57 & -46 & 0 & 12.2 & 19.90 & 0.10 & 0.65 & 2.719 & -26.05 & 1 & & 0 \\
\hline 21 & 31 & 14.89 & -46 & 56 & 9.1 & 19.26 & -0.30 & 0.37 & 0.980 & -24.56 & 1 & & 0 \\
\hline 21 & 31 & 9.87 & -43 & 39 & 29.7 & 20.76 & -0.33 & 0.65 & 1.390 & -23.79 & 1 & & 0 \\
\hline 21 & 31 & 15.15 & -45 & 7 & 35.1 & 19.19 & -0.73 & 0.52 & 1.966 & -26.09 & 1 & & 0 \\
\hline 21 & 31 & 15.80 & -45 & 1 & 19.0 & 19.67 & -0.22 & 1.15 & 0.418 & -22.32 & 0 & & 3 \\
\hline 21 & 31 & 21.94 & -46 & 38 & 23.6 & 18.71 & -0.45 & 0.54 & 1.720 & -26.29 & 1 & 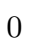 & 0 \\
\hline 21 & 31 & 15.90 & -42 & 43 & 18.9 & 20.20 & 0.08 & 0.99 & 0.365 & -21.50 & 1 & & 0 \\
\hline 21 & 31 & 22.52 & -43 & 43 & 53.5 & 20.55 & -0.41 & 0.70 & 0.844 & -22.95 & 1 & & 0 \\
\hline 21 & 31 & 26.19 & -45 & 15 & 3.1 & 20.69 & -0.72 & 1.12 & 1.920 & -24.54 & 1 & & 3 \\
\hline 21 & 31 & 27.03 & -45 & 4 & 49.3 & 19.85 & 0.00 & 1.12 & 0.715 & -23.29 & 1 & & 0 \\
\hline 21 & 31 & 23.41 & -42 & 59 & 60.0 & 20.13 & -0.39 & 0.80 & 1.648 & -24.78 & 1 & & 0 \\
\hline 21 & 31 & 37.75 & -45 & 48 & 53.2 & 20.65 & -0.20 & 1.37 & 0.781 & -22.68 & 0 & & 3 \\
\hline 21 & 31 & 35.99 & -44 & 10 & 45.4 & 21.12 & -0.62 & 1.25 & 0.529 & -21.38 & 0 & & 3 \\
\hline 21 & 31 & 33.39 & -42 & 57 & 51.0 & 18.22 & -0.67 & 0.74 & 2.096 & -27.19 & 1 & & 0 \\
\hline 21 & 31 & 43.86 & -46 & 57 & 13.0 & 17.97 & 0.03 & 0.54 & 0.684 & -25.08 & 1 & & 0 \\
\hline 21 & 31 & 36.21 & -44 & 3 & 24.2 & 20.35 & -0.36 & 0.63 & 1.660 & -24.58 & 0 & & 0 \\
\hline 21 & 31 & 36.77 & -43 & 39 & 43.3 & 19.90 & -0.35 & 0.74 & 0.843 & -23.59 & 0 & & 0 \\
\hline 21 & 31 & 38.88 & -43 & 54 & 22.2 & 20.40 & -0.34 & 0.42 & 1.216 & -23.87 & 0 & & 0 \\
\hline 21 & 31 & 38.93 & -42 & 47 & 29.3 & 20.39 & -0.36 & 1.23 & 0.982 & -23.43 & 1 & & 3 \\
\hline 21 & 31 & 43.86 & -43 & 39 & 11.8 & 20.52 & -0.53 & 0.55 & 0.715 & -22.62 & 0 & 2 & 0 \\
\hline 21 & 31 & 50.98 & -43 & 19 & 14.5 & 20.05 & -0.38 & 0.51 & 1.656 & -24.87 & 0 & & 0 \\
\hline 21 & 31 & 54.25 & -44 & 29 & 23.0 & 19.14 & -0.49 & 0.50 & 1.414 & -25.45 & 1 & & 0 \\
\hline 21 & 32 & 2.95 & -45 & 16 & 9.7 & 18.91 & -0.55 & 0.79 & 1.890 & -26.29 & 1 & & 0 \\
\hline 21 & 31 & 56.70 & -42 & 55 & 39.3 & 21.08 & -0.36 & 1.70 & 1.730 & -23.93 & 0 & 2 & 3 \\
\hline 21 & 32 & 10.46 & -46 & 14 & 20.6 & 19.60 & 0.34 & 0.72 & 2.769 & -26.39 & 1 & & 0 \\
\hline 21 & 32 & 4.70 & -42 & 45 & 41.3 & 19.02 & -0.61 & 0.70 & 1.992 & -26.29 & 1 & & 0 \\
\hline 21 & 32 & 13.49 & -45 & 16 & 50.0 & 17.68 & -0.59 & 0.55 & 0.507 & -24.72 & 1 & & 0 \\
\hline 21 & 32 & 13.00 & -44 & 14 & 14.0 & 19.65 & -0.22 & 0.63 & 1.645 & -25.26 & 1 & 2 & 0 \\
\hline 21 & 32 & 11.55 & -43 & 24 & 45.8 & 19.87 & -0.35 & 1.71 & 0.860 & -23.67 & 1 & 2 & 3 \\
\hline 21 & 32 & 18.25 & -45 & 6 & 59.8 & 19.20 & -0.45 & 0.61 & 0.520 & -23.26 & 1 & & 0 \\
\hline 21 & 32 & 18.33 & -44 & 18 & 48.4 & 19.87 & -0.35 & 0.97 & 1.451 & -24.77 & 1 & & 0 \\
\hline 21 & 32 & 21.72 & -43 & 24 & 47.5 & 20.43 & -0.11 & 0.52 & 2.240 & -25.12 & 1 & U & 0 \\
\hline 21 & 32 & 23.85 & -43 & 48 & 10.8 & 20.19 & 0.23 & 0.86 & 2.790 & -25.81 & 1 & 0 & 0 \\
\hline 21 & 32 & 23.30 & -43 & 9 & 42.2 & 20.82 & -0.56 & 0.45 & 0.959 & -22.95 & 0 & 2 & 0 \\
\hline 21 & 32 & 27.48 & -44 & 5 & 24.8 & 20.41 & -0.28 & 0.98 & 0.478 & -21.87 & 1 & & 0 \\
\hline 21 & 32 & 36.44 & -46 & 34 & 0.5 & 19.46 & -0.53 & 1.33 & 1.320 & -24.99 & 1 & & 3 \\
\hline 21 & 32 & 35.27 & -46 & 11 & 31.5 & 19.01 & -0.46 & 0.66 & 1.600 & -25.84 & 1 & 2 & 0 \\
\hline 21 & 32 & 26.58 & -43 & 18 & 4.3 & 19.66 & -0.31 & 0.82 & 1.710 & -25.33 & 1 & 2 & 0 \\
\hline 21 & 32 & 31.07 & -44 & 18 & 57.9 & 19.08 & -0.77 & 1.25 & 1.258 & -25.26 & 1 & 2 & 3 \\
\hline 21 & 32 & 37.49 & -45 & 8 & 31.6 & 20.12 & -0.56 & 0.59 & 1.377 & -24.41 & 1 & & 0 \\
\hline 21 & 32 & 37.63 & -44 & 51 & 1.2 & 18.55 & -0.41 & 0.91 & 0.920 & -25.13 & 1 & 0 & 0 \\
\hline 21 & 32 & 38.00 & -44 & 20 & 41.8 & 20.61 & -0.53 & 1.12 & 0.655 & -22.34 & 1 & 2 & 0 \\
\hline 21 & 32 & 54.14 & -47 & 5 & 8.9 & 19.38 & -0.44 & 1.59 & 0.244 & -21.44 & 1 & 0 & 3 \\
\hline 21 & 32 & 53.63 & -45 & 58 & 49.2 & 20.29 & -0.41 & 0.76 & 1.003 & -23.57 & 1 & 2 & 0 \\
\hline 21 & 32 & 51.16 & -44 & 31 & 17.9 & 21.33 & -0.09 & 1.76 & 0.430 & -20.72 & 0 & & 3 \\
\hline 21 & 33 & 3.03 & -46 & 20 & 24.7 & 20.87 & 0.27 & 0.78 & 2.760 & -25.11 & 1 & 0 & 0 \\
\hline 21 & 32 & 55.05 & -43 & 21 & 44.1 & 17.93 & 0.27 & 0.48 & 2.420 & -27.78 & 1 & 0 & 0 \\
\hline
\end{tabular}




\begin{tabular}{|c|c|c|c|c|c|c|c|c|c|c|c|c|c|}
\hline \multicolumn{3}{|c|}{ R.A. (1950) } & \multicolumn{3}{|c|}{ Dec (1950) } & \multirow{2}{*}{$\begin{array}{c}B \\
20.06\end{array}$} & \multirow{2}{*}{$\begin{array}{l}U-B \\
-0.29\end{array}$} & \multirow{2}{*}{$\begin{array}{c}\delta m \\
0.28\end{array}$} & \multirow{2}{*}{$\begin{array}{c}z \\
0.327\end{array}$} & \multirow{2}{*}{$\begin{array}{c}M_{B} \\
-21.40\end{array}$} & \multicolumn{3}{|c|}{ Samples } \\
\hline 21 & 33 & 2.60 & -44 & 16 & 33.7 & & & & & & 0 & 2 & 0 \\
\hline 21 & 33 & 6.66 & -44 & 28 & 26.5 & 20.80 & -0.68 & 0.99 & 0.544 & -21.76 & & & 0 \\
\hline 21 & 33 & 7.60 & -44 & 16 & 50.7 & 20.76 & 9.99 & 0.75 & 3.340 & -25.61 & & & 0 \\
\hline 21 & 33 & 13.11 & -45 & 20 & 2.1 & 20.65 & 0.34 & 0.49 & 2.898 & -25.43 & & & 0 \\
\hline 21 & 33 & 7.84 & -43 & 4 & 13.9 & 20.25 & 0.19 & 1.00 & 2.470 & -25.50 & & & 0 \\
\hline 21 & 33 & 12.34 & -42 & 58 & 51.0 & 19.40 & -0.22 & 0.43 & 1.190 & -24.83 & & & 0 \\
\hline 21 & 33 & 22.63 & -44 & 20 & 23.7 & 19.96 & -0.37 & 0.45 & 0.999 & -23.90 & & & 0 \\
\hline 21 & 33 & 31.09 & -46 & 11 & 38.3 & 19.72 & -0.37 & 0.65 & 0.888 & -23.89 & & & 0 \\
\hline 21 & 33 & 31.37 & -46 & 13 & 34.8 & 19.58 & -0.44 & 1.36 & 1.448 & -25.06 & & & 3 \\
\hline 21 & 33 & 21.32 & -43 & 30 & 55.6 & 21.29 & -0.55 & 0.92 & 1.720 & -23.71 & & & 0 \\
\hline 21 & 33 & 23.97 & -43 & 36 & 17.3 & 20.42 & -0.20 & 0.46 & 0.669 & -22.58 & & & 0 \\
\hline 21 & 33 & 29.69 & -44 & 4 & 8.7 & 20.37 & -0.22 & 0.90 & 1.756 & -24.67 & & & 0 \\
\hline 21 & 33 & 39.37 & -46 & 3 & 3.6 & 20.75 & 0.03 & 1.45 & 0.423 & -21.26 & & & 3 \\
\hline 21 & 33 & 26.75 & -42 & 55 & 1.3 & 20.45 & -0.14 & 1.57 & 0.580 & -22.24 & & & 3 \\
\hline 21 & 33 & 38.98 & -45 & 34 & 51.6 & 19.44 & -0.24 & 1.54 & 0.868 & -24.12 & & & 3 \\
\hline 21 & 33 & 41.36 & -45 & 53 & 0.8 & 18.34 & -0.54 & 0.36 & 1.000 & -25.52 & & & 0 \\
\hline 21 & 33 & 51.84 & -46 & 17 & 51.0 & 20.75 & -0.49 & 1.21 & 1.571 & -24.06 & & & 0 \\
\hline 21 & 33 & 53.98 & -46 & 17 & 24.2 & 19.65 & -0.40 & 0.85 & 0.844 & -23.85 & & & 0 \\
\hline 21 & 33 & 58.56 & -47 & 8 & 7.8 & 19.35 & -0.62 & 1.10 & 1.097 & -24.70 & & 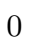 & 3 \\
\hline 21 & 33 & 45.77 & -43 & 58 & 44.9 & 18.27 & -0.33 & 0.83 & 1.560 & -26.53 & L & & 0 \\
\hline 21 & 33 & 45.15 & -43 & 49 & 23.8 & 20.09 & -0.59 & 0.77 & 1.850 & -25.06 & & & 0 \\
\hline 21 & 33 & 47.57 & -44 & 17 & 35.9 & 20.35 & 0.44 & 0.45 & 2.838 & -25.69 & & & 0 \\
\hline 21 & 33 & 48.75 & -44 & 28 & 48.7 & 20.21 & -0.51 & 0.51 & 2.059 & -25.17 & & & 0 \\
\hline 21 & 34 & 1.71 & -46 & 46 & 47.6 & 19.90 & 0.63 & 0.74 & 3.065 & -26.29 & & 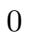 & 0 \\
\hline 21 & 33 & 53.80 & -44 & 56 & 27.5 & 20.08 & 0.03 & 0.62 & 2.745 & -25.89 & & & 0 \\
\hline 21 & 33 & 46.40 & -43 & 21 & 34.2 & 20.24 & -0.69 & 0.83 & 2.022 & -25.10 & & & 0 \\
\hline 21 & 33 & 51.02 & -43 & 6 & 10.0 & 20.81 & -0.55 & 0.85 & 1.400 & -23.76 & & & 0 \\
\hline 21 & 34 & 8.30 & -45 & 6 & 3.0 & 20.59 & -0.31 & 0.53 & 0.880 & -23.00 & 1 & 2 & 0 \\
\hline 21 & 34 & 2.22 & -43 & 10 & 38.6 & 21.12 & -0.50 & 0.56 & 0.524 & -21.36 & 0 & & 0 \\
\hline 21 & 34 & 8.13 & -43 & 51 & 26.6 & 19.56 & -0.24 & 1.03 & 1.663 & -25.37 & . & & 0 \\
\hline 21 & 34 & 19.95 & -46 & 2 & 17.9 & 20.41 & -0.65 & 1.52 & 1.292 & -23.99 & & & 3 \\
\hline 21 & 34 & 8.81 & -43 & 11 & 33.7 & 19.92 & -0.24 & 0.74 & 1.650 & -24.99 & 1 & 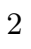 & 0 \\
\hline 21 & 34 & 19.94 & -45 & 16 & 58.6 & 20.44 & -0.48 & 0.84 & 1.106 & -23.63 & 1 & 2 & 0 \\
\hline 21 & 34 & 17.90 & -43 & 50 & 8.8 & 19.88 & -0.35 & 0.82 & 1.558 & -24.91 & L & 2 & 0 \\
\hline 21 & 34 & 13.93 & -42 & 57 & 43.8 & 19.30 & 0.11 & 0.60 & 2.608 & -26.56 & 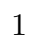 & & 0 \\
\hline 21 & 34 & 18.64 & -43 & 52 & 39.7 & 20.71 & -0.61 & 0.64 & 1.370 & -23.81 & & 2 & 0 \\
\hline 21 & 34 & 37.07 & -46 & 45 & 4.8 & 19.37 & -0.23 & 0.82 & 0.704 & -23.74 & 1 & 0 & 0 \\
\hline 21 & 34 & 30.32 & -44 & 35 & 55.7 & 20.27 & -0.72 & 1.48 & 1.319 & -24.17 & 0 & 0 & 3 \\
\hline 21 & 34 & 29.90 & -44 & 3 & 5.5 & 20.47 & -0.12 & 1.11 & 1.576 & -24.35 & L & 0 & 0 \\
\hline 21 & 34 & 47.85 & -46 & 2 & 39.1 & 20.15 & -0.52 & 0.56 & 1.340 & -24.33 & 0 & 2 & 0 \\
\hline 21 & 34 & 50.90 & -46 & 1 & 49.4 & 17.81 & 0.12 & 0.46 & 0.528 & -24.68 & 1 & U & 0 \\
\hline 21 & 34 & 37.41 & -43 & 40 & 37.4 & 19.39 & -0.25 & 0.48 & 0.837 & -24.09 & 0 & 2 & 0 \\
\hline 21 & 34 & 49.15 & -44 & 43 & 0.2 & 19.53 & 0.15 & 0.64 & 2.526 & -26.27 & 1 & 0 & 0 \\
\hline 21 & 35 & 0.95 & -46 & 11 & 36.5 & 18.87 & -0.41 & 0.27 & 1.903 & -26.34 & 0 & & 0 \\
\hline 21 & 34 & 43.15 & -43 & 6 & 56.5 & 19.67 & -0.36 & 0.81 & 1.423 & -24.93 & & 2 & 0 \\
\hline 21 & 35 & 6.63 & -46 & 32 & 27.6 & 18.66 & -0.08 & 1.02 & 2.222 & -26.87 & 1 & 0 & 0 \\
\hline 21 & 35 & 2.90 & -45 & 49 & 32.5 & 20.06 & -0.59 & 0.72 & 1.832 & -25.07 & 1 & 2 & 0 \\
\hline 21 & 35 & 4.13 & -45 & 47 & 3.5 & 20.36 & -0.46 & 1.15 & 1.845 & -24.79 & 0 & 2 & 0 \\
\hline 21 & 35 & 0.77 & -44 & 16 & 28.6 & 20.67 & -0.27 & 0.26 & 0.285 & -20.49 & & 2 & 0 \\
\hline 21 & 34 & 56.25 & -43 & 13 & 47.4 & 20.08 & -0.30 & 0.85 & 2.141 & -25.38 & & 2 & 0 \\
\hline 21 & 35 & 19.47 & -46 & 20 & 47.4 & 18.41 & -0.21 & 0.53 & 0.505 & -23.99 & 1 & 0 & 0 \\
\hline 21 & 35 & 0.16 & -43 & 4 & 14.3 & 19.60 & -0.26 & 1.96 & 0.511 & -22.82 & 1 & 2 & 3 \\
\hline 21 & 35 & 4.58 & -43 & 46 & 13.0 & 20.87 & 0.50 & 0.59 & 3.100 & -25.35 & & U & 0 \\
\hline 21 & 35 & 14.93 & -45 & 16 & 31.0 & 20.94 & -0.20 & 1.21 & 0.655 & -22.01 & & 2 & 3 \\
\hline 21 & 35 & 5.14 & -43 & 3 & 48.9 & 19.62 & -0.45 & 0.47 & 2.029 & -25.72 & 1 & 2 & 0 \\
\hline
\end{tabular}




\begin{tabular}{|c|c|c|c|c|c|c|c|c|c|c|c|c|c|}
\hline \multicolumn{3}{|c|}{ R.A. (1950) } & \multicolumn{3}{|c|}{ Dec (1950) } & \multirow{2}{*}{$\begin{array}{c}B \\
19.30\end{array}$} & \multirow{2}{*}{$\begin{array}{l}U-B \\
-0.33\end{array}$} & \multirow{2}{*}{$\begin{array}{l}\delta m \\
0.34\end{array}$} & \multirow{2}{*}{$\begin{array}{c}z \\
0.929\end{array}$} & \multirow{2}{*}{$\begin{array}{c}M_{B} \\
-24.40\end{array}$} & \multicolumn{3}{|c|}{ Samples } \\
\hline 21 & 35 & 12.41 & -44 & 2 & 15.0 & & & & & & 0 & 2 & 0 \\
\hline 21 & 35 & 28.41 & -45 & 59 & 2.1 & 19.95 & -0.56 & 0.72 & 0.568 & -22.70 & 1 & & 0 \\
\hline 21 & 35 & 13.86 & -43 & 24 & 17.3 & 19.18 & -0.42 & 0.77 & 1.935 & -26.07 & 1 & & 0 \\
\hline 21 & 35 & 28.59 & -45 & 29 & 56.1 & 20.77 & -0.27 & 0.64 & 2.148 & -24.69 & 1 & & 0 \\
\hline 21 & 35 & 28.35 & -45 & 23 & 57.7 & 18.69 & -0.47 & 0.66 & 1.097 & -25.36 & 1 & & 0 \\
\hline 21 & 35 & 22.69 & -44 & 7 & 50.2 & 20.89 & -0.36 & 0.75 & 1.168 & -23.30 & 1 & & 0 \\
\hline 21 & 35 & 20.13 & -42 & 53 & 29.7 & 17.89 & -0.45 & 0.64 & 1.469 & -26.78 & 1 & & 0 \\
\hline 21 & 35 & 23.26 & -43 & 20 & 7.1 & 20.33 & -0.33 & 1.00 & 1.535 & -24.43 & 1 & & 0 \\
\hline 21 & 35 & 34.64 & -43 & 49 & 30.6 & 21.12 & -0.54 & 0.86 & 1.395 & -23.44 & 0 & & 0 \\
\hline 21 & 35 & 41.40 & -44 & 0 & 49.2 & 18.45 & -0.32 & 0.87 & 0.461 & -23.75 & 0 & & 0 \\
\hline 21 & 35 & 38.64 & -43 & 26 & 25.7 & 20.65 & 0.06 & 0.46 & 2.483 & -25.11 & 1 & & 0 \\
\hline 21 & 35 & 43.33 & -44 & 8 & 11.1 & 20.59 & -0.48 & 1.23 & 1.785 & -24.49 & 0 & & 0 \\
\hline 21 & 35 & 47.12 & -44 & 1 & 23.2 & 20.41 & -0.18 & 0.60 & 1.708 & -24.58 & 1 & & 0 \\
\hline 21 & 35 & 43.26 & -43 & 20 & 54.9 & 20.38 & -0.11 & 0.55 & 2.186 & -25.12 & 1 & & 0 \\
\hline 21 & 36 & 6.35 & -46 & 26 & 0.5 & 19.48 & -0.37 & 1.31 & 0.920 & -24.20 & 0 & & 3 \\
\hline 21 & 35 & 53.01 & -44 & 21 & 44.3 & 18.69 & -0.15 & 0.70 & 0.408 & -23.25 & 1 & & 0 \\
\hline 21 & 35 & 54.17 & -44 & 14 & 32.4 & 21.14 & -0.51 & 0.27 & 0.412 & -20.82 & 0 & & 0 \\
\hline 21 & 35 & 54.42 & -43 & 45 & 24.2 & 20.47 & -0.43 & 0.98 & 1.633 & -24.42 & 1 & & 0 \\
\hline 21 & 36 & 19.27 & -46 & 48 & 48.0 & 18.88 & -0.11 & 0.54 & 1.124 & -25.23 & 1 & & 0 \\
\hline 21 & 36 & 17.27 & -44 & 30 & 11.6 & 19.80 & -0.46 & 0.41 & 0.931 & -23.91 & 0 & & 0 \\
\hline 21 & 36 & 28.18 & -45 & 38 & 4.6 & 19.14 & -0.22 & 0.59 & 1.695 & -25.83 & 1 & & 0 \\
\hline 21 & 36 & 21.01 & -43 & 17 & 51.2 & 20.29 & -0.39 & 0.98 & 1.048 & -23.67 & 0 & & 0 \\
\hline 21 & 36 & 31.16 & -44 & 20 & 53.9 & 21.14 & -0.35 & 0.29 & 0.433 & -20.92 & 0 & 2 & 0 \\
\hline 21 & 36 & 28.71 & -43 & 47 & 29.6 & 18.96 & -0.34 & 0.61 & 1.520 & -25.78 & 1 & & 0 \\
\hline 21 & 36 & 30.45 & -43 & 1 & 22.7 & 18.12 & -0.57 & 0.62 & 1.343 & -26.36 & 1 & & 0 \\
\hline 21 & 36 & 39.18 & -44 & 11 & 35.1 & 19.63 & -0.41 & 0.69 & 1.925 & -25.61 & 1 & & 0 \\
\hline 21 & 36 & 52.42 & -45 & 29 & 1.7 & 19.43 & -0.09 & 0.75 & 1.174 & -24.77 & 1 & & 0 \\
\hline 21 & 36 & 34.83 & -42 & 48 & 0.0 & 20.10 & 0.08 & 0.40 & 0.168 & -19.92 & 1 & U & 0 \\
\hline 21 & 37 & 4.86 & -46 & 20 & 11.6 & 19.22 & -0.02 & 0.52 & 2.495 & -26.55 & 1 & & 0 \\
\hline 21 & 36 & 42.71 & -43 & 30 & 28.1 & 20.86 & -0.33 & 1.41 & 1.028 & -23.06 & 1 & & 3 \\
\hline 21 & 36 & 49.17 & -44 & 20 & 47.4 & 20.23 & -0.30 & 0.35 & 0.275 & -20.85 & 0 & & 0 \\
\hline 21 & 36 & 42.52 & -43 & 28 & 46.6 & 18.38 & -0.43 & 0.54 & 0.250 & -22.50 & 1 & 2 & 0 \\
\hline 21 & 36 & 59.31 & -45 & 17 & 26.5 & 20.67 & -0.38 & 0.86 & 1.105 & -23.40 & 1 & & 0 \\
\hline 21 & 36 & 44.29 & -42 & 51 & 36.2 & 19.67 & -0.41 & 0.78 & 1.510 & -25.06 & 1 & & 0 \\
\hline 21 & 37 & 8.20 & -45 & 23 & 36.4 & 20.45 & -0.38 & 0.94 & 1.645 & -24.46 & 1 & & 0 \\
\hline 21 & 37 & 2.10 & -44 & 22 & 56.2 & 20.64 & -0.52 & 0.75 & 2.050 & -24.73 & 0 & 2 & 0 \\
\hline 21 & 37 & 12.13 & -45 & 20 & 33.6 & 20.70 & -0.34 & 0.58 & 2.113 & -24.73 & 0 & 2 & 0 \\
\hline 21 & 37 & 6.81 & -43 & 54 & 27.7 & 19.70 & -0.77 & 0.75 & 1.915 & -25.52 & 1 & & 0 \\
\hline 21 & 37 & 25.87 & -45 & 29 & 27.9 & 20.33 & -0.39 & 0.60 & 1.438 & -24.30 & 1 & & 0 \\
\hline 21 & 37 & 14.45 & -44 & 6 & 39.4 & 20.30 & -0.05 & 0.62 & 2.250 & -25.26 & 1 & U & 0 \\
\hline 21 & 37 & 17.79 & -44 & 21 & 10.6 & 19.72 & -0.35 & 1.42 & 0.346 & -21.86 & 1 & 2 & 3 \\
\hline 21 & 37 & 17.76 & -44 & 10 & 44.8 & 20.87 & -0.42 & 0.65 & 0.798 & -22.51 & 0 & 2 & 0 \\
\hline 21 & 37 & 10.59 & -43 & 5 & 26.6 & 20.92 & -0.42 & 1.32 & 1.393 & -23.64 & 1 & & 3 \\
\hline 21 & 37 & 29.30 & -44 & 49 & 49.2 & 19.54 & 0.49 & 0.72 & & -20.02 & 1 & & 0 \\
\hline 21 & 37 & 15.41 & -43 & 1 & 47.3 & 19.83 & -0.22 & 1.11 & 1.520 & -24.91 & 1 & 2 & 3 \\
\hline 21 & 37 & 31.36 & -44 & 22 & 18.3 & 19.42 & 0.32 & 0.54 & 1.320 & -25.03 & 1 & 0 & 0 \\
\hline 21 & 37 & 53.50 & -46 & 45 & 21.6 & 20.42 & 0.24 & 1.11 & 2.800 & -25.59 & 1 & 0 & 0 \\
\hline 21 & 37 & 32.47 & -43 & 36 & 56.0 & 19.53 & -0.10 & 0.48 & 2.310 & -26.08 & 1 & U & 0 \\
\hline 21 & 37 & 38.02 & -43 & 43 & 5.3 & 19.16 & -0.74 & 0.50 & 1.964 & -26.12 & 1 & 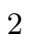 & 0 \\
\hline 21 & 38 & 0.90 & -46 & 11 & 10.3 & 19.48 & 0.04 & 0.66 & 2.287 & -26.11 & 1 & 0 & 0 \\
\hline 21 & 37 & 51.68 & -44 & 35 & 48.8 & 18.34 & -0.34 & 0.35 & 0.632 & -24.54 & 1 & 0 & 0 \\
\hline 21 & 37 & 45.95 & -43 & 56 & 50.9 & 20.02 & -0.63 & 1.67 & 2.039 & -25.34 & 0 & 2 & 3 \\
\hline 21 & 38 & 17.41 & -46 & 35 & 7.3 & 21.02 & -0.37 & 1.40 & 0.780 & -22.31 & 0 & & 3 \\
\hline 21 & 38 & 15.98 & -46 & 21 & 31.8 & 19.45 & -0.16 & 0.61 & 0.618 & -23.38 & 1 & 0 & 0 \\
\hline 21 & 38 & 16.48 & -45 & 12 & 28.1 & 19.34 & -0.24 & 0.25 & 2.164 & -26.14 & 0 & 2 & 0 \\
\hline
\end{tabular}


M.R.S. Hawkins: Quasar variability: Correlations with amplitude

\begin{tabular}{|c|c|c|c|c|c|c|c|c|c|c|c|c|c|}
\hline \multicolumn{3}{|c|}{ R.A. (1950) } & \multicolumn{3}{|c|}{ Dec (1950) } & \multirow{2}{*}{$\begin{array}{c}B \\
19.66\end{array}$} & \multirow{2}{*}{$\begin{array}{l}U-B \\
-0.59\end{array}$} & \multirow{2}{*}{$\begin{array}{c}\delta m \\
0.51\end{array}$} & \multirow{2}{*}{$\begin{array}{c}z \\
2.102\end{array}$} & \multirow{2}{*}{$\begin{array}{c}M_{B} \\
-25.76\end{array}$} & \multicolumn{3}{|c|}{ Samples } \\
\hline 21 & 38 & 11.93 & -44 & 20 & 28.7 & & & & & & 1 & 2 & 0 \\
\hline 21 & 38 & 5.54 & -43 & 20 & 26.1 & 19.91 & -0.17 & 0.54 & 0.574 & -22.76 & 1 & 0 & 0 \\
\hline 21 & 38 & 41.58 & -46 & 50 & 33.2 & 18.26 & -0.20 & 1.02 & 0.762 & -25.02 & 1 & 0 & 0 \\
\hline 21 & 38 & 14.85 & -43 & 45 & 49.7 & 19.26 & -0.48 & 0.93 & 1.320 & -25.19 & 1 & 2 & 0 \\
\hline 21 & 38 & 12.87 & -43 & 9 & 10.4 & 20.22 & -0.17 & 2.08 & 0.687 & -22.84 & 1 & 0 & 3 \\
\hline 21 & 38 & 25.45 & -44 & 5 & 15.3 & 19.85 & -0.31 & 0.73 & 0.862 & -23.69 & 1 & 2 & 0 \\
\hline 21 & 38 & 15.73 & -42 & 55 & 44.8 & 20.13 & -0.23 & 0.83 & 1.597 & -24.72 & 1 & 2 & 0 \\
\hline 21 & 38 & 33.14 & -44 & 16 & 15.2 & 20.53 & -0.33 & 1.73 & 0.623 & -22.32 & 0 & 0 & \\
\hline 21 & 38 & 28.59 & -43 & 7 & 4.9 & 20.50 & -0.30 & 0.26 & 0.255 & -20.42 & 0 & 2 & 0 \\
\hline 21 & 38 & 43.40 & -44 & 32 & 17.9 & 0.00 & -0.51 & 1.44 & 1.660 & -44.93 & 0 & 0 & 3 \\
\hline 21 & 38 & 31.80 & -43 & 13 & 51.2 & 20.06 & -0.75 & 0.79 & 1.287 & -24.33 & 1 & 2 & 0 \\
\hline 21 & 38 & 56.08 & -45 & 26 & 4.1 & 20.08 & -0.51 & 0.36 & 1.197 & -24.16 & 1 & 2 & 0 \\
\hline 21 & 38 & 56.93 & -45 & 16 & 15.5 & 20.50 & -0.50 & 1.50 & 2.013 & -24.83 & 0 & 2 & \\
\hline 21 & 39 & 0.08 & -45 & 20 & 38.8 & 20.01 & -0.20 & 0.64 & 2.210 & -25.51 & 1 & 2 & 0 \\
\hline 21 & 38 & 53.72 & -44 & 38 & 39.4 & 19.50 & -0.53 & 0.61 & 1.078 & -24.52 & 1 & 0 & 0 \\
\hline 21 & 39 & 12.24 & -46 & 14 & 18.8 & 20.15 & 1.14 & 0.59 & 3.340 & -26.22 & 1 & 0 & 0 \\
\hline 21 & 38 & 57.01 & -44 & 16 & 52.4 & 19.31 & -0.37 & 0.62 & 1.735 & -25.71 & 1 & 2 & 0 \\
\hline 21 & 38 & 54.54 & -43 & 41 & 29.9 & 20.74 & -0.33 & 1.02 & 2.210 & -24.78 & 1 & 2 & 0 \\
\hline 21 & 38 & 59.14 & -43 & 50 & 57.6 & 18.71 & 0.21 & 0.40 & 0.142 & -20.94 & 1 & 0 & 0 \\
\hline 21 & 38 & 52.05 & -42 & 58 & 42.7 & 20.26 & -0.46 & 0.56 & 0.937 & -23.46 & 1 & 2 & 0 \\
\hline 21 & 38 & 54.79 & -42 & 55 & 28.0 & 19.87 & -0.20 & 1.18 & 0.542 & -22.68 & 1 & 2 & 3 \\
\hline 21 & 39 & 25.50 & -45 & 20 & 14.0 & 19.97 & -0.69 & 0.79 & 1.394 & -24.59 & 1 & 2 & 0 \\
\hline 21 & 39 & 20.18 & -44 & 43 & 60.0 & 20.96 & 0.09 & 0.77 & 2.380 & -24.71 & 1 & 0 & 0 \\
\hline 21 & 39 & 27.52 & -45 & 21 & 30.5 & 20.73 & -0.24 & 0.79 & 1.671 & -24.21 & 1 & 2 & 0 \\
\hline 21 & 39 & 9.07 & -42 & 54 & 48.2 & 20.62 & -0.32 & 0.73 & 1.905 & -24.59 & 0 & 2 & 0 \\
\hline 21 & 39 & 22.45 & -43 & 32 & 4.6 & 18.40 & 0.11 & 0.61 & 2.190 & -27.10 & 1 & 0 & 0 \\
\hline 21 & 39 & 32.94 & -43 & 55 & 45.4 & 19.47 & -0.51 & 0.64 & 0.679 & -23.56 & 1 & 2 & 0 \\
\hline 21 & 39 & 44.37 & -44 & 49 & 37.2 & 20.10 & -0.14 & 1.46 & 1.640 & -24.80 & 0 & 0 & 3 \\
\hline 21 & 39 & 27.24 & -43 & 8 & 28.0 & 20.86 & -0.20 & 1.04 & 0.752 & -22.39 & 1 & 2 & 0 \\
\hline 21 & 40 & 3.43 & -46 & 20 & 2.2 & 18.78 & -0.22 & 0.65 & 0.548 & -23.79 & 1 & 0 & 0 \\
\hline 21 & 39 & 30.84 & -42 & 59 & 26.0 & 19.81 & -0.35 & 0.59 & 1.890 & -25.39 & 1 & 2 & 0 \\
\hline 21 & 39 & 42.04 & -43 & 28 & 17.4 & 20.55 & -0.29 & 0.85 & 0.708 & -22.57 & 0 & 2 & 0 \\
\hline 21 & 39 & 48.87 & -43 & 36 & 18.4 & 20.86 & -0.56 & 1.21 & 1.379 & -23.68 & 0 & 0 & 3 \\
\hline 21 & 40 & 16.52 & -45 & 52 & 37.0 & 18.11 & -0.34 & 0.71 & 1.688 & -26.85 & 1 & 0 & 0 \\
\hline 21 & 39 & 51.98 & -43 & 14 & 14.2 & 21.21 & -0.15 & 1.48 & 1.645 & -23.70 & 0 & 0 & 3 \\
\hline
\end{tabular}

